
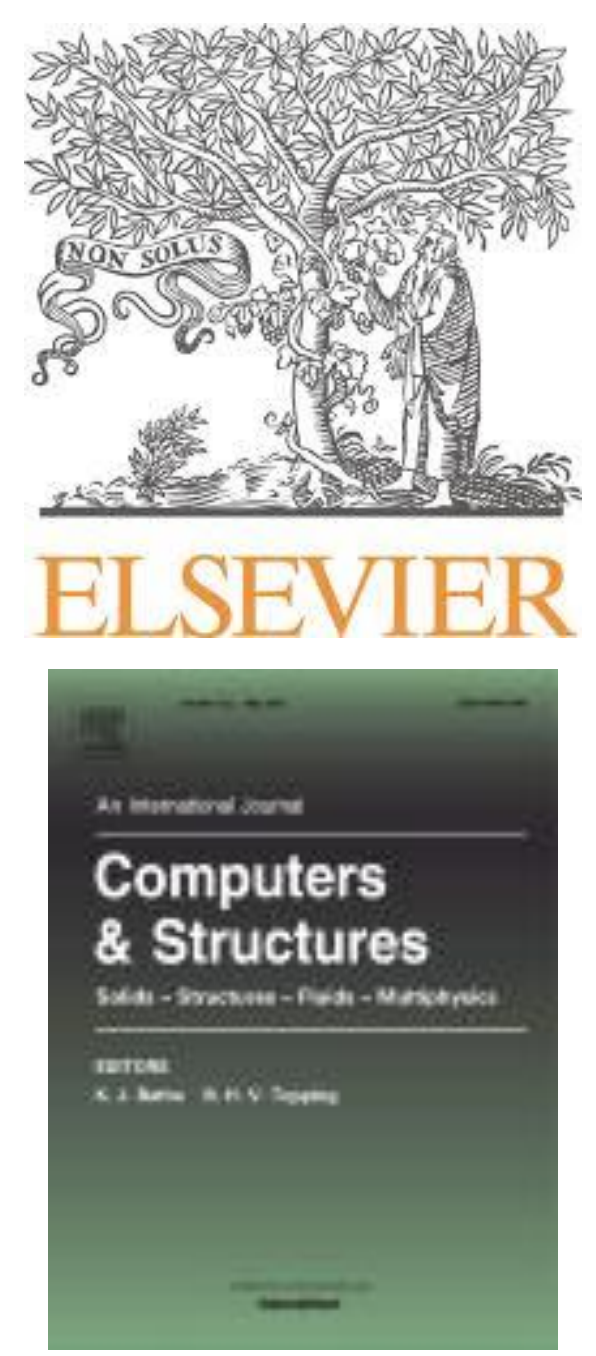

This is a pre-print version of an article appeared in a journal published by Elsevier. When citing, please refer to the published version:

B. D'Amico, A. Kermani, H. Zhang, P. Shepherd, C.J.K. Williams, Optimization of cross-section of actively bent grid shells with strength and geometric compatibility constraints, Computers and Structures, 154 (2015) 163-176.

DOI: http://doi.org/10.1016/i.compstruc.2015.04.006 


\title{
Optimisation of cross-section of actively bent grid shells with strength and geometric compatibility constraints
}

\author{
B. D’Amico ${ }^{\mathrm{a}, *}$, A. Kermani ${ }^{\mathrm{a}}$, H. Zhang ${ }^{\mathrm{a}}$, P. Shepherd ${ }^{\mathrm{b}}$, C. J. K. Williams ${ }^{\mathrm{b}}$ \\ ${ }^{a}$ Centre for Timber Engineering (CTE), School of Engineering and the Built Environment, \\ Edinburgh Napier University, Edinburgh, UK \\ ${ }^{b}$ Department of Architecture and Civil Engineering, University of Bath, Bath, UK
}

\begin{abstract}
The use of bending as self forming process allows the realization of shape-resistant systems, such as grid shell structures. Here, a numerical method for optimisation of the cross-section of actively bent structures is introduced. For a given load distribution, the optimisation objective consists of normalizing the bending stresses to a given value on the entire structure. In addition, strength and geometric compatibility constraints are taken into account. The method is demonstrated by numerical examples. Further, in order to handle the large displacements involved, a co-rotational Finite Element formulation is adopted and modified to take into account the changes in stiffness that occur in the forming process of active bending systems. The modified co-rotational formulation is solved for static equilibrium using a Dynamic Relaxation scheme, and is tested against the analytical solutions of some preliminary test cases, as well as experimental results, and shown to be 'accurate'.
\end{abstract}

Keywords: Active Bending, Grid Shell, Structural Optimisation, Co-rotational Formulation, Dynamic Relaxation, Timber Structures

\section{Introduction}

The term 'Active Bending' defines a category of structural systems in which bending is used as a self-forming process [1]. For instance, the realization of grid

\footnotetext{
${ }^{*}$ Corresponding author, telephone: +44 (0) 1314552249

Email addresses: bernardinodamico@gmail.com, b.d'amico@napier.ac.uk (B. D'Amico), a.kermani@napier.ac.uk (A. Kermani), j.zhang@napier.ac.uk (H. Zhang), p.shepherd@bath.ac.uk (P. Shepherd), c.j.k.williams@bath.ac.uk (C. J. K. Williams) 
shell systems obtained by assembling an initially flat mat made of continuous elastic

5

rods (e.g fibre reinforced polymers [2] or timber $[3,4,5,6,7,8]$ ) and successive forming by means of adjustable scaffolding or temporary crane-cable systems. Shell systems derive their strength and stiffness from their inherent doubly curved shape, allowing them to work mainly in membrane action under the effect of external loads. Nevertheless, a certain amount of out-of-plane stiffness is required to resist inextentional deformations [9].

The double-layer technique, first adopted in the design of the Mannheim timber grid shell for the Garden Festival [3] allows tighter curvatures to be obtained compared to a single-layer mat made from rods with equivalent cross-sectional area. Once the forming process is complete, sliding between overlapping laths is constrained by inserting shear blocks in between the laths making up the single rib (see Figure 1) thus enhancing the out-of-plane bending stiffness of the equivalent continuous shell.

The shape of such (actively bent) grid shell systems can be modelled by performing a preliminary simulation of the forming process by means of non-linear finite element procedures. Thus the resulting geometry can then be used as a basis for further structural analyses. Nevertheless, the effect of residual pre-stress forces on the overall structural behaviour, as well as the change in stiffness due to the presence of shear blocks, needs to be taken into account when assessing the actual load-carrying capacity of the structure.

A comprehensive numerical procedure is introduced here to solve the initial form finding phase, the construction process simulation and successive load calculations of such actively bent grid shell systems. A modified co-rotational beam element with six degrees of freedom (DoF), in conjunction with the Dynamic Relaxation method (DR), allows the change in stiffness of the post-formed mat to be taken into account whilst, maintaining the resulting equilibrium configuration of the doublelayer mat with sliding connections. Consequently, an optimisation method for deriving the double-layer cross-section is proposed. For a given load configuration, the iterative method allows the bending stress ratios to be 'consolidated', resulting in a grid shell geometry with members having variable cross-section. Practical issues, rising from the fact of having a different cross-section for each member, can be handled by post-rationalizing members into groups, or providing fabrication's methods that allow to 'accurately' reproduce the linear variation of each member's 
profile. Further discussion about this will be addressed in the conclusions with a prospective from the structural point of view.

(a)

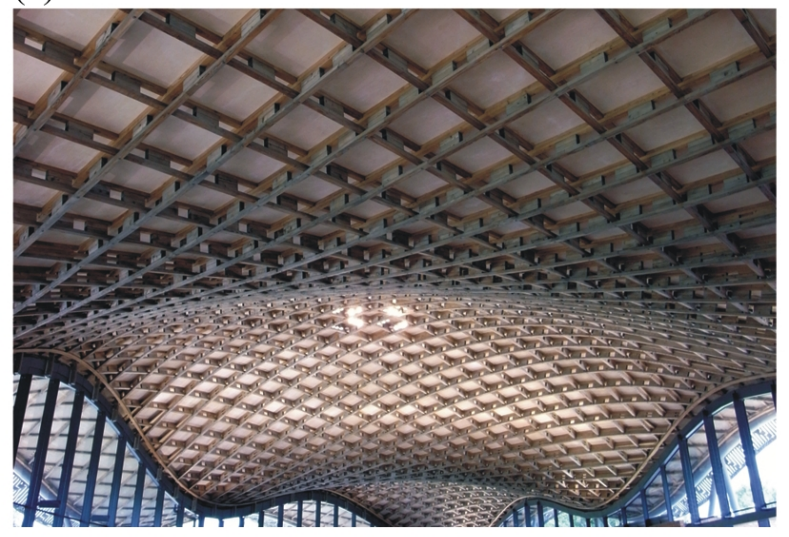

(b)

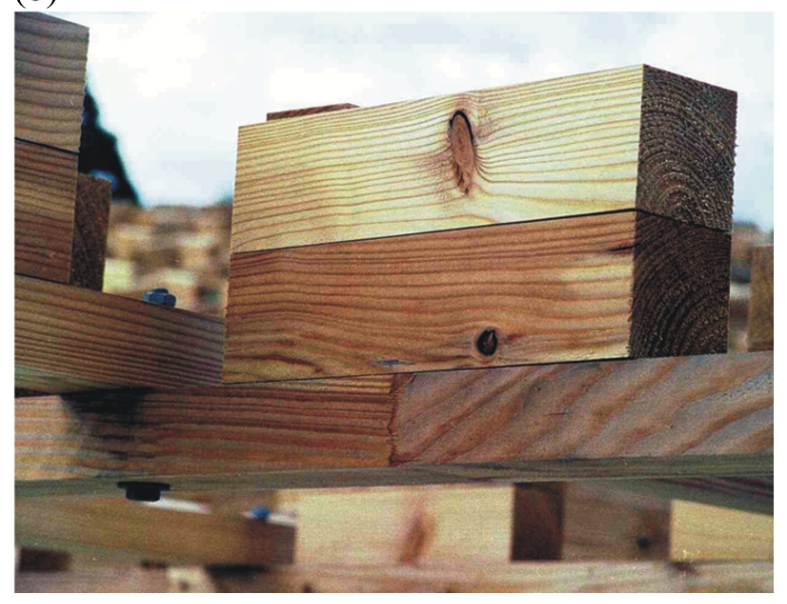

Figure 1: Savill Garden grid shell, Windsor, UK 2006 [10]: (a) Internal view; (b) Detail of the shear block connection. (Photos courtesy - Richard Harris)

\section{Preliminary Theory}

\subsection{Co-rotational formulation}

In order to handle the large displacements and rotations involved in the form finding process of actively bent structures, a co-rotational formulation [11, 12] for a three-dimensional beam element is adopted. Unlike the Total Lagrangian and 
Updated Lagrangian formulations [13], in the co-rotational approach the motion of the element is treated as a result of a rigid motion plus a deformation.

Assuming a geometry represented by a discrete set of nodes $\mathbf{P}$ with coordinate $\bar{p}_{i}$ with arbitrary initial position in the Cartesian coordinate system:

$$
\mathbf{P}=\left\{\bar{p}_{1} \ldots \bar{p}_{i} \ldots \bar{p}_{m^{\circ}}\right\} \quad ; \quad \bar{p}_{i}=\left[\begin{array}{lll}
x & y & z
\end{array}\right]
$$

and a connectivity list $\mathbf{E}$ storing the nodes' indices of the element ends $(1,2)$ :

$$
\mathbf{E}=\left\{\mathbf{e}_{1} \ldots \mathbf{e}_{j} \ldots \mathbf{e}_{n^{\circ}}\right\} \quad ; \quad \mathbf{e}_{j}=\left\{i_{1}, i_{2}\right\}
$$

the rigid motion of the $j$ th element is determined by an auxiliary vector $\bar{p}_{j}$ connecting the element end nodes $\left(\bar{p}_{i_{1}}, \bar{p}_{i_{2}}\right)$. Then, assuming a 'right-handed' local reference frame $\left\{\bar{x}_{i}, \bar{y}_{i}, \bar{z}_{i}\right\}$ for the generic $i$ node, the element deformation (local rotational and axial displacements) is determined by computing the local frame orientation of end nodes with respect to the element vector position $\bar{p}$.
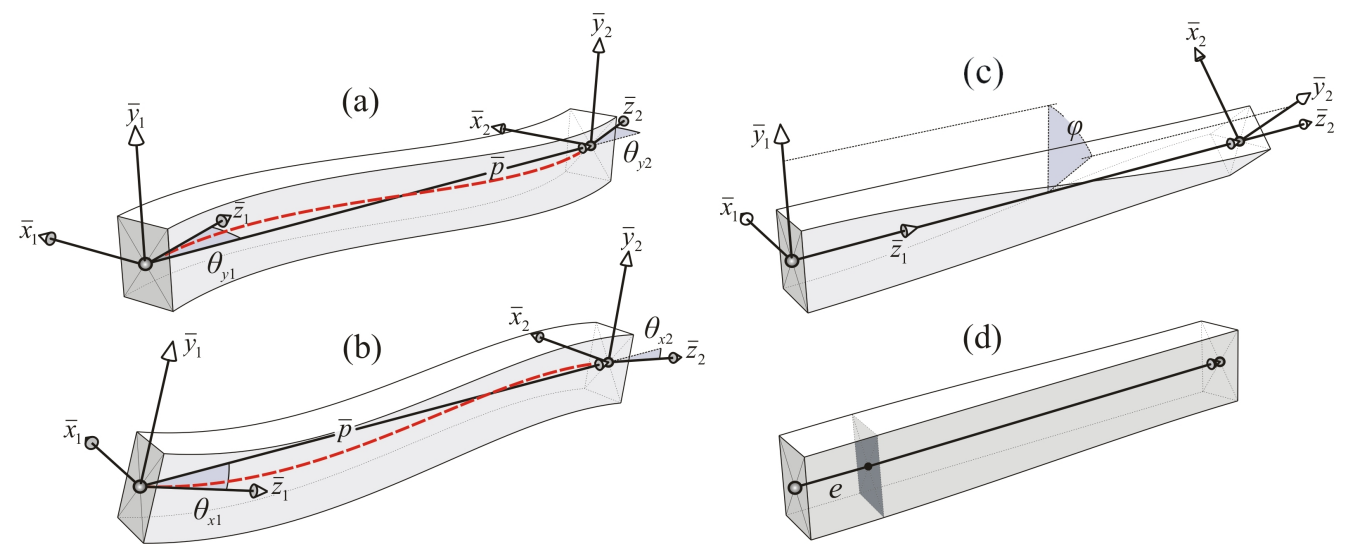

Figure 2: Co-rotational formulation for a three-dimensional beam element: (a) Rotations around the local $\bar{y}$ axes; (b) Rotations around the local $\bar{x}$ axes; (c) Angle of twist; (d) Axial shortening/elongation; The dashed line represents the element's cubic shape function.

With reference to Figure 2, the local rotations of the $\bar{e}_{j}$ element around the local $\left(\bar{x}_{i}\right.$ and $\left.\bar{y}_{i}\right)$ axes at its start node $i_{1}$ are $\theta_{x, 1}$ and $\theta_{y, 1}$, while $\theta_{x, 2}$ and $\theta_{y, 2}$ are the rotations around (the local frame) at its $i_{2}$ end node. Whereas, $\varphi$ is the angle of twist while $e$ is the axial shortening/elongation. The local shear displacements are not explicitly set out because of the reference axes choice (at a nodal level instead of element level). 
From the element's local rotations and displacements, the corresponding element's ends reactions can be obtained by differentiating the beam's expression of total strain energy $U$ [14] thus obtaining the bending moments $M_{x, 1}, M_{y, 1}, M_{x, 2}$ and $M_{y, 2}$, the torsion moment $M_{\varphi}$ and the axial force $N$. Again, the local shear forces are missing due to the reference axes choice. Such an element's local reactions are a function of its material and geometric stiffness i.e.: the second moments of area $\left(I_{x}\right.$ and $\left.I_{y}\right)$, torsional constant $(J)$, cross-sectional area $(A)$, element's unstressed length $\left(L_{0}\right)$, Young's and shear moduli $(E$ and $G)$ :

$$
\begin{gathered}
N=\frac{E A}{L_{0}} e \quad ; \quad M_{\varphi}=\frac{G J}{L_{0}} \varphi \\
M_{x, 1}=\frac{N L_{0}}{30}\left(4 \theta_{x, 1}-\theta_{x, 2}\right)+\frac{2 E I_{x}}{L_{0}}\left(2 \theta_{x, 1}+\theta_{x, 2}\right)
\end{gathered}
$$

70

$$
\begin{aligned}
& M_{x, 2}=\frac{N L_{0}}{30}\left(4 \theta_{x, 2}-\theta_{x, 1}\right)+\frac{2 E I_{x}}{L_{0}}\left(2 \theta_{x, 2}+\theta_{x, 1}\right) \\
& M_{y, 1}=\frac{N L_{0}}{30}\left(4 \theta_{y, 1}-\theta_{y, 2}\right)+\frac{2 E I_{y}}{L_{0}}\left(2 \theta_{y, 1}+\theta_{y, 2}\right) \\
& M_{y, 2}=\frac{N L_{0}}{30}\left(4 \theta_{y, 2}-\theta_{y, 1}\right)+\frac{2 E I_{y}}{L_{0}}\left(2 \theta_{y, 2}+\theta_{y, 1}\right)
\end{aligned}
$$

The element's bowing effect is taken into account by the appearance of the axial force term $N$ in the equations of moment (4 - 7). The local element's end scalar reactions so found are then transformed into global vector reactions forces by imposing static equilibrium to the element [15] or assuming equivalence of strain energy [16] thus obtaining the global shear force vector components (missing at a local reference frame level). With the global element's end reactions so found, an out-of-balance force $\bar{R}_{i}$ and out-of-balance moment $\bar{H}_{i}$ can be calculated for the generic $i$ th node as vector summation of global reactions of the elements surrounding the node, plus external applied forces (and moments). Accordingly, the equilibrium geometry (nodes position and local frame orientations) such that the residuals $\bar{R}_{i}$ and $\bar{H}_{i}$ are null, can be found by implementation of a explicit resolution method such as Dynamic Relaxation (DR). The resolution of co-rotational beamelement formulation by DR method was first developed by Williams (as reported by Adriaenssens [14]). 


\subsubsection{The Dynamic Relaxation}

The DR method, firstly proposed by Day [17] and Otter [18], is a fictitious timestepping scheme, where the positions of nodes representing a structural system are obtained by iterative numerical integration of Newton's second law of motion until the entire system reaches static equilibrium by the application of a viscous or kinetic [19] damping term. For a given structural system, the finding of the equilibrium geometry such that the residual forces and moments $\left(\bar{R}_{i}\right.$ and $\left.\bar{H}_{i}\right)$ are null, can be pursued by implicit Finite Element analysis procedures (e.g. the well known Newton-Raphson method). However, an explicit Finite Element approach (such as DR in conjunction with the co-rotational formulation) allows the solution to converge independently of the magnitude of the initial deformed state, and thus is more suitable for form finding analyses involving large displacements. Moreover, since the DR operates at a vector level, it does not require the assembly and manipulation of a global stiffness matrix, hence it is relatively easy to implement and suitable for parallel computing [20]. In order to increase numerical stability and the size of the time step, a 4th order Runge-Kutta method (RK4) [21] is adopted for the numerical integration of the translational and rotational acceleration terms, thus briefly described as follow:

Assuming, for the $i$ th node, the vector position $\bar{p}_{i}$ as given in the second of Eqs. (1) and the corresponding acceleration and velocity terms (respectively $\bar{a}_{i}$ and $\bar{v}_{i}$ ) as:

$$
\bar{a}_{i}=\left[\begin{array}{lll}
\ddot{x} & \ddot{y} & \ddot{z}
\end{array}\right] \quad ; \quad \bar{v}_{i}=\left[\begin{array}{lll}
\dot{x} & \dot{y} & \dot{z}
\end{array}\right]
$$

the node's velocity at the time $(t+\Delta t)$ is computed from the acceleration and velocity terms at time $t$ :

$$
\begin{aligned}
& \bar{k}_{1}=\bar{a}_{i}^{t}=\frac{1}{m_{i}} \bar{R}_{i}^{t} \\
& \bar{k}_{2}=\bar{a}_{i}^{t}+\frac{\Delta t}{2} \bar{k}_{1} \\
& \bar{k}_{3}=\bar{a}_{i}^{t}+\frac{\Delta t}{2} \bar{k}_{2} \\
& \bar{k}_{4}=\bar{a}_{i}^{t}+\Delta t \bar{k}_{3} \\
& \bar{v}_{i}^{t+\Delta t}=c \bar{v}_{i}^{t}+\frac{\Delta t}{6}\left(\bar{k}_{1}+2 \bar{k}_{2}+2 \bar{k}_{3}+\bar{k}_{4}\right)
\end{aligned}
$$

with $m=$ 'fictitious' lumped nodal mass and $c=$ viscous damping factor $\in[0,1]$. 
Accordingly, by assuming this time:

$$
\begin{aligned}
& \bar{k}_{1}=\bar{v}_{i}^{t+\Delta t} \\
& \bar{k}_{2}=\bar{v}_{i}^{t+\Delta t}+\frac{\Delta t}{2} \bar{k}_{1} \\
& \bar{k}_{3}=\bar{v}_{i}^{t+\Delta t}+\frac{\Delta t}{2} \bar{k}_{2} \\
& \bar{k}_{4}=\bar{v}_{i}^{t+\Delta t}+\Delta t \bar{k}_{3}
\end{aligned}
$$

the updated vector position at time $(t+\Delta t)$ is given by:

$$
\bar{p}_{i}^{t+\Delta t}=\bar{p}_{i}^{t}+\frac{\Delta t}{6}\left(\bar{k}_{1}+2 \bar{k}_{2}+2 \bar{k}_{3}+\bar{k}_{4}\right)
$$

In the same way, indicating for the $i$ th node, the pseudo vectors of angular acceleration and angular velocity as:

$$
\bar{a}_{i}=\left[\begin{array}{lll}
\ddot{\vartheta}_{x} & \ddot{\vartheta}_{y} & \ddot{\vartheta}_{z}
\end{array}\right] \quad ; \quad \bar{v}_{i}=\left[\begin{array}{lll}
\dot{\vartheta}_{x} & \dot{\vartheta}_{y} & \dot{\vartheta}_{z}
\end{array}\right]
$$

the pseudo vector of rotations $\bar{\vartheta}_{i}$ (to not be confused with the element's local rotations) is:

$$
\bar{\vartheta}_{i}=\left[\begin{array}{lll}
\vartheta_{x} & \vartheta_{y} & \vartheta_{z}
\end{array}\right]
$$

Such pseudo vector contains the rotation angles of the local frame $\left\{\bar{x}_{i}, \bar{y}_{i}, \bar{z}_{i}\right\}$ around the global directions $x, y$ and $z$, and can be computed by applying again Eqs. (9) and (10) but substituting (in the first of Eqs. (9)) the nodal out of balance force $\bar{R}_{i}$ with the out-of-balance moment $\bar{H}_{i}$ and the fictitious lumped mass $m$ with the fictitious lumped moment of inertia (rotational mass) therefore obtaining:

$$
\bar{\vartheta}_{i}^{t+\Delta t}=\frac{\Delta t}{6}\left(\bar{k}_{1}+2 \bar{k}_{2}+2 \bar{k}_{3}+\bar{k}_{4}\right)
$$

The rotation angles $\vartheta_{x}, \vartheta_{y}$ and $\vartheta_{z}$ obtained by Eq. (14) are non-additive (and non-commutative) quantities, hence they cannot be updated in the same way as for the translational displacements, which is why the recurrence Eq. (11) provides an 'absolute' coordinate value, whilst Eq. (14) only provides an 'increments' of rotations (of the local $i$ th frame) around the global directions. The local frame orientation $\left\{\bar{x}_{i}, \bar{y}_{i}, \bar{z}_{i}\right\}^{t}$ is then updated to $\left\{\bar{x}_{i}, \bar{y}_{i}, \bar{z}_{i}\right\}^{t+\Delta t}$ by pre-multiplying each unit vector with a rotation matrix, which is only as function of the previously found pseudo vector $\bar{\vartheta}_{i}^{t+\Delta t}$ as described in [15]. 


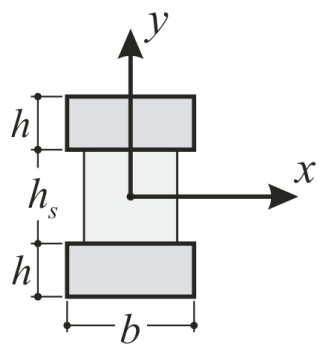

Figure 3: Built-up cross-section.

\subsection{Modelling double-layer systems}

The double layer technique allows tighter curvatures to be obtained compared to a single layer mat made of laths with equivalent cross-sectional area. Once the forming process is complete, sliding between overlapping laths is constrained (thus, enhancing the bending stiffness of the built-up rib) by inserting timber shear blocks in between the laths making up the single rib. Accordingly, in order to perform load analyses continuously during the forming (construction) process, the change in bending stiffness due to the presence of shear blocks needs to be taken into account. For a built-up member subject to bending due to external loads, the slip between overlapped laths leads to a discontinuity of strains at the interface, resulting in a difference in curvature of the individual laths. Consequently, a 'correct' numerical model should consider a single element for each overlapping lath in order to fully model the mechanical behaviour of the composite member. 'An alternative, slightly less accurate method' [22] assumes compatibility in the displacements and curvatures at the interface of overlapped laths, by considering the fasteners of the shear block connections as a series of linear springs having shear stiffness $(K)$, therefore modelling the built-up member as a single element assuming an 'equivalent' $E I$ value as a function of the shear stiffness of the spring.

Since during the forming process, sliding between overlapping laths is allowed, the values of cross-sectional area and second moment of area, used for the corotational beam-model, are twice that of the corresponding single lath value (see Figure 3):

$$
I_{x}^{*}=b h^{3} / 6
$$

Then, for a load analysis, the increase of bending stiffness due to presence of shear blocks needs to be taken into account. Assuming an infinitely rigid connection 
between laths and shear blocks (absence of slip at the interfaces), the resulting second moment of area around the local $\bar{x}$ axis is:

$$
I_{x}^{* *}=\frac{b\left(2 h+h_{s}\right)^{3}}{12}-\frac{b h_{s}^{3}}{12}
$$

On the other hand, assuming the contribution to stiffness given by shear blocks as null, the resulting second moment of area is obviously that given by Eq. (15). Therefore, by considering that:

$$
\begin{gathered}
I_{x}^{* *}=\frac{b\left(2 h+h_{s}\right)^{3}}{12}-\frac{b h_{s}^{3}}{12}=\frac{b}{6}\left(4 h^{3}+6 h^{2} h_{s}+3 h h_{s}^{2}\right)= \\
=\frac{4}{6} b h^{3}+\frac{b}{6}\left(6 h^{2} h_{s}+3 h h_{s}^{2}\right)=\frac{1}{6} b h^{3}+\frac{3}{6} b h^{3}+\frac{b}{6}\left(6 h^{2} h_{s}+3 h h_{s}^{2}\right)= \\
=\frac{b h^{3}}{6}+\left(\frac{h^{2}}{2}+\frac{h_{s}^{2}}{2}+h h_{s}\right) b h
\end{gathered}
$$

a general equation can be arranged:

$$
I_{x}=c_{s} \frac{\left(h+h_{s}\right)^{2}}{2} b h+\frac{b h^{3}}{6} \quad ; \quad c_{s} \in[0,1]
$$

where, $c_{s}$ is set to zero to simulate the forming process, while a value $>0$ is set for load analyses in order to take into account the increase in bending stiffness due to the presence of shear blocks. The connection efficiency factor $c_{s}$ of Eq. (18) will be a function of the elastic modulus, cross-sectional area $(A=b h)$, rod's length $(L)$, horizontal shear spring stiffness $(K)$ and springs (fastener) spacing $(s)$ :

$$
c_{s}=f(E, b, h, L, K, s)
$$

Where: for $K \approx \infty$ (e.g. glued connection) then $c_{s}=1$ while, for $K=0$ (e.g. no shear blocks) $\Rightarrow c_{s}=0$. For instance, according to Eq. B.5 in Annex B of Eurocode 5 (EC5) [23]:

$$
c_{s}=\left[1+\pi^{2} E A s /\left(K L^{2}\right)\right]^{-1}
$$

Noting that EC5 Eq. (20) is based on the assumption that the element is pin jointed at its ends. 


\subsection{Modified force-displacement relations}

Setting up the numerical model by assuming an equivalent $E I$, it can be stated that for preliminary numerical simulations of the forming process, a value $c_{s}=0$ is applied, while for successive load analyses, a $c_{s}>0$ is applied (obtained e.g. by Eq. (20)) in order to take into account the increase in bending stiffness due to the presence of shear blocks.

Nevertheless, when setting the updated second moment of area to perform the successive (load) analysis, the change in stiffness generates unbalance forces. In other words, the system searches for the equilibrium configuration that would have resulted by forming the double-layer mat with shear blocks in place at the flat configuration. In order to maintain the equilibrium of the stress field obtained when the forming process is complete (with $I_{x}^{c_{s}=0}$ ) the corresponding element end reactions $M_{x, 1}, M_{x, 2}$ as from Eqs. (4 - 5) must maintain the equilibrium values $\left(M_{x, 1}^{e q .}, M_{x, 2}^{e q .}\right)$ regardless of the new $I_{x}^{c_{s}>0}$ value. Therefore, the angular rotations $\theta_{x, 1}^{e q .}$ and $\theta_{x, 2}^{e q .}$ need to be multiplied by a reduction factor corresponding to the ratio $\left(I_{x}^{c_{s}=0} / I_{x}^{c_{s}>0}\right)[15]$.

The difference between the local rotation angles at form finding equilibrium $\theta_{x}^{e q}$. and the reduced values are:

$$
\theta_{x, 1}^{e q .}\left(1-\frac{I_{x}^{c_{s}=0}}{I_{x}^{c_{s}>0}}\right) \quad ; \quad \theta_{x, 2}^{e q .}\left(1-\frac{I_{x}^{c_{s}=0}}{I_{x}^{c_{s}>0}}\right)
$$

From a physical point of view, such differences can be conceived as the element's unstressed local rotations that would result if the bent rods were re straightened leaving the shear blocks inserted. It is easy to imagine that in doing such an operation, the double layer mat would not recover the flat configuration anymore. In addition, the reduction of pre-stress forces due to material (e.g. wood) relaxation can be modelled by introducing a reduction factor $c_{R} \in[0,1]$ such that the unstressed local rotation angles become:

$$
\begin{aligned}
& \theta_{x, 1}^{e q .}\left(1-\frac{I_{x}^{c_{s}=0}}{I_{x}^{c_{s}>0}} c_{R}\right) ;\left(1-c_{R}\right) \theta_{y, 1}^{e q .} \\
& \theta_{x, 2}^{e q .}\left(1-\frac{I_{x}^{c_{s}=0}}{I_{x}^{c_{s}>0}} c_{R}\right) ;\left(1-c_{R}\right) \theta_{y, 2}^{e q .}
\end{aligned}
$$

Accordingly, by subtracting at each time increment the unstressed local rotation angles (Eqs. (22)) from the current element's local rotations, the force-displacements 
Eqs. (4 - 7) become:

$$
\begin{gathered}
M_{x, 1}=\frac{N L_{0}}{30}\left(4 \theta_{x, 1}-\theta_{x, 2}\right)+ \\
+\frac{2 E I_{x}^{c_{s}>0}}{L_{0}}\left\{2\left[\theta_{x, 1}-\theta_{x, 1}^{e q .}\left(1-\frac{I_{x}^{c_{s}=0}}{I_{x}^{c_{s}>0}} c_{R}\right)\right]+\left[\theta_{x, 2}-\theta_{x, 2}^{e q .}\left(1-\frac{I_{x}^{c_{s}=0}}{I_{x}^{c_{s}>0}} c_{R}\right)\right]\right\} \\
M_{x, 2}=\frac{N L_{0}}{30}\left(4 \theta_{x, 2}-\theta_{x, 1}\right)+ \\
+\frac{2 E I_{x}^{c_{s}>0}}{L_{0}}\left\{2\left[\theta_{x, 2}-\theta_{x, 2}^{e q .}\left(1-\frac{I_{x}^{c_{s}=0}}{I_{x}^{c_{s}>0}} c_{R}\right)\right]+\left[\theta_{x, 1}-\theta_{x, 1}^{e q .}\left(1-\frac{I_{x}^{c_{s}=0}}{I_{x}^{c_{s}>0}} c_{R}\right)\right]\right\}
\end{gathered}
$$

200

$$
\begin{gathered}
M_{y, 1}=\frac{N L_{0}}{30}\left(4 \theta_{y, 1}-\theta_{y, 2}\right)+ \\
+\frac{2 E I_{y}}{L_{0}}\left\{2\left[\theta_{y, 1}-\theta_{y, 1}^{e q .}\left(1-c_{R}\right)\right]+\left[\theta_{y, 2}-\theta_{y, 2}^{e q .}\left(1-c_{R}\right)\right]\right\} \\
M_{y, 2}=\frac{N L_{0}}{30}\left(4 \theta_{y, 2}-\theta_{y, 1}\right)+ \\
+\frac{2 E I_{y}}{L_{0}}\left\{2\left[\theta_{y, 2}-\theta_{y, 2}^{e q .}\left(1-c_{R}\right)\right]+\left[\theta_{y, 1}-\theta_{y, 1}^{e q .}\left(1-c_{R}\right)\right]\right\}
\end{gathered}
$$

Eqs. (23 - 26) will be used for load analyses performed as a continuity of the simulation of the forming process. From these, can be seen that:

- For $c_{R}=0 \Rightarrow$ the local rotation angles at form finding equilibrium $\theta^{\text {eq. }}$ correspond to the unstressed local rotations, or in simpler words, the geometry at completion of the forming process is stress-free (material fully relaxed).

- For $c_{R}=1 \Rightarrow$ the terms $\theta_{y, 1}^{e q .}$ and $\theta_{y, 2}^{e q}$ in Eqs. (25 - 26) disappear, meaning that the bending pre-stress due to the forming process is fully present. Nevertheless, a 'non-null' component of the unstressed local rotations (as from Eqs. (21)) around the local $\bar{x}$ axis is still present in Eqs. (23 - 24) as a result of the shear blocks insertion.

Although limited to the linear-elastic case, the described formulation can be straightforwardly extended to simulate more realistic material's behaviour such as 
non-linear elasticity and elastic-plastic behaviour [35, 36] (i.e. by introduction of plastic hinges during the analysis). In fact, provided a stable mass/time-step ratio, explicit methods are insensitive to discontinuities of the stress-strain relationship (i.e. due to the material non-linearity) in converging to the numerical solution.

\subsubsection{Stress field}

On the basis of the co-rotational beam element formulation given in subsection 2.1, the distribution of normal stress $\sigma$ at the external fibres of the beam crosssection along the element can be obtained from the curvature values $\kappa_{x}$ and $\kappa_{y}$. By deriving the Hermite cubic shape function $\bar{p}(t)$ with respect to the parameter $t \in[0,1]$ representing the relative position along the element, thus $\bar{p}(0)=\bar{p}_{i_{1}}$ and $\bar{p}(1)=\bar{p}_{i_{2}}$, the curvature functions $\kappa_{x}(t)$ and $\kappa_{y}(t)$ around the element local axes $\operatorname{are}^{1}[14]$ :

$$
\begin{gathered}
\kappa_{x}(t)=\frac{(6 t-2) \theta_{x, 1}+(6 t-4) \theta_{x, 2}}{|\bar{p}|} \\
\kappa_{y}(t)=-\frac{\left[(6 t-2) \theta_{y, 1}+(6 t-4) \theta_{y, 2}\right]}{|\bar{p}|}
\end{gathered}
$$

where $|\bar{p}|$ is modulus of the auxiliary vector connecting the element's end nodes (to not be confused with the node's vector position). As previously done for the loaddisplacement functions, by subtracting at each time increment the unstressed local rotation angles from the current element's local rotations, the curvature functions (Eqs. (27)) become:

$$
\begin{gathered}
\kappa_{x}(t)=\frac{1}{|\bar{p}|}\left\{(6 \mathbf{t}-\mathbf{c}) \cdot\left[\boldsymbol{\theta}_{x}-\left(1-c_{R}\right) \boldsymbol{\theta}_{x}^{e q \cdot}\right]\right\} \\
\kappa_{y}(t)=-\frac{1}{|\bar{p}|}\left\{(6 \mathbf{t}-\mathbf{c}) \cdot\left[\boldsymbol{\theta}_{y}-\left(1-c_{R}\right) \boldsymbol{\theta}_{y}^{e q \cdot}\right]\right\}
\end{gathered}
$$

where:

$$
\mathbf{t}=\left[\begin{array}{l}
t \\
t
\end{array}\right] \quad ; \quad \mathbf{c}=\left[\begin{array}{l}
2 \\
4
\end{array}\right] \quad ; \quad \boldsymbol{\theta}_{x}=\left[\begin{array}{l}
\theta_{x, 1} \\
\theta_{x, 2}
\end{array}\right] \quad ; \quad \boldsymbol{\theta}_{y}=\left[\begin{array}{l}
\theta_{y, 1} \\
\theta_{y, 2}
\end{array}\right]
$$

235 Noting that:

For $c_{R}=0 \Rightarrow$ the curvatures generated by the forming process correspond to the unstressed curvature values (stress-free geometry).

\footnotetext{
${ }^{1} \operatorname{In}[14] \kappa_{x}(t)$ and $\kappa_{y}(t)$ are parametrized assuming $t \in[-0.5,+0.5]$.
} 
For $c_{R}=1 \Rightarrow$ Eqs. (27) and (28) give the same result (geometry fully prestressed).
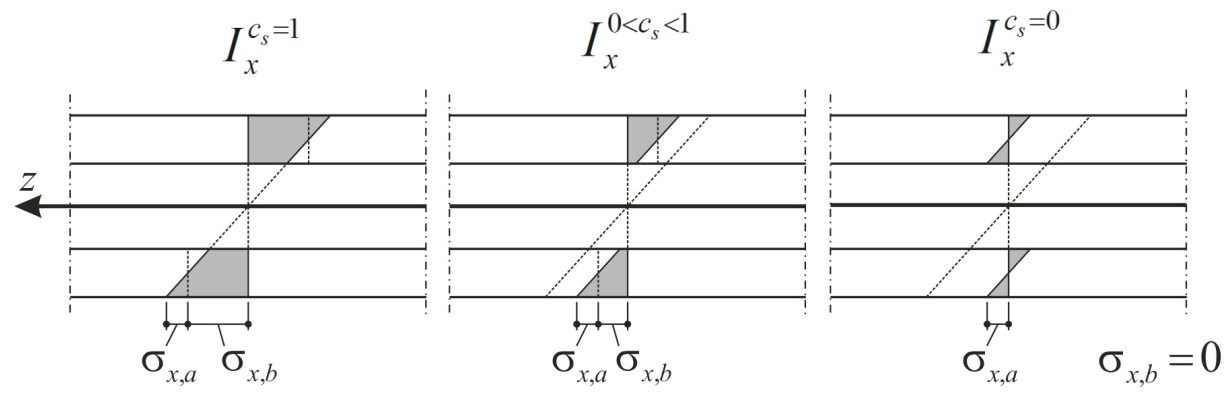

Figure 4: Built-up cross-section: Normal stress distribution $\left(\sigma_{x}\right)$ for different values of the connection efficiency factor $\left(c_{s}\right)$.

Accordingly, assuming the bending stress as:

$$
\sigma(t)=\sigma_{x}(t)+\sigma_{y}(t)
$$

the bending stress $\sigma_{y}$ can be obtained from the second of Eqs. (28) as:

$$
\sigma_{y}(t)=\frac{b E \kappa_{y}(t)}{2}
$$

while $\sigma_{x}$ will be a function of $\kappa_{x}(t)$ plus some other term due to the presence of shear blocks. With reference to Figure 4:

$$
\sigma_{x}(t)=\sigma_{x, a}+\sigma_{x, b}=\frac{h E \kappa_{x}(t)}{2}+\frac{N_{s}(t)}{b h}
$$

where $N_{s}(t)$ is obtained from the following lever-arm relation:

$$
N_{s}(t)=\frac{M_{x, b}(t)}{\left(h+h_{s}\right)}
$$

and $M_{x, b}(t)$ computed considering only the contribution to $E I_{x}$ due to shear blocks (only the first term of Eq. (18)):

$$
M_{x, b}(t)=E I_{x, b} \kappa_{x}(t) \quad ; \quad I_{x, b}=c_{s} \frac{\left(h+h_{s}\right)^{2}}{2} b h
$$

Since the moment contribution $M_{x, b}(t)$ and stress contribution $\sigma_{x, b}$ (both) due to shear blocks only occur after the grid shell is formed (bent); for $\theta_{x, 1}=\theta_{x, 1}^{e q}$ and $\theta_{x, 2}=\theta_{x, 2}^{e q .} \Rightarrow M_{x, b}(t)$ and $\sigma_{x, b}$ must be null. Therefore $\kappa_{x}(t)$ in Eq. (34) is 
calculated by setting $c_{R}=0$ in Eq. (28) regardless of the $c_{R}$ value used to compute the element reaction forces (Eqs. $(23-26))$ and the bending stress $\sigma_{x, a}$.

Accordingly:

$$
M_{x, b}(t)=E c_{s} b h \frac{\left(h+h_{s}\right)^{2}}{2|\bar{p}|}\left[(6 \mathbf{t}-\mathbf{c}) \cdot\left(\boldsymbol{\theta}_{x}-\boldsymbol{\theta}_{x}^{\text {eq. }}\right)\right]
$$

with t, c and $\boldsymbol{\theta}_{x}$ as given in Eqs. (29). Substituting then Eq. (35) into Eq. (33) and (32):

$$
\sigma_{x, b}(t)=E c_{s} \frac{\left(h+h_{s}\right)}{2|\bar{p}|}\left[(6 \mathbf{t}-\mathbf{c}) \cdot\left(\boldsymbol{\theta}_{x}-\boldsymbol{\theta}_{x}^{e q .}\right)\right]
$$

Hence, Eq. (32) becomes:

$$
\begin{gathered}
\sigma_{x}(t)=\frac{E}{2|\bar{p}|}\left\{\left(h c_{s}+h_{s} c_{s}+h\right)\left[\left(\theta_{x, 1}-\theta_{x, 1}^{e q .}\right)(6 t-2)+\left(\theta_{x, 2}-\theta_{x, 2}^{e q .}\right)(6 t-4)\right]+\right. \\
\left.+h c_{R}\left[\theta_{x, 1}^{e q .}(6 t-2)+\theta_{x, 2}^{e q .}(6 t-4)\right]\right\}
\end{gathered}
$$

\subsubsection{Shear blocks strength ratios}

In order to verify the shear block connections, the shear reaction force $T_{s}$ generated by the blocks can be obtained as the finite difference of the variation of axial force $N_{s}(t)$ :

$$
T_{s}=N_{s}(t=1)-N_{s}(t=0)
$$

From Eqs. (33) and (35):

$$
T_{s}=E c_{s} b h \frac{3\left(h+h_{s}\right)}{|\bar{p}|}\left(\theta_{x, 1}-\theta_{x, 1}^{e q .}+\theta_{x, 2}-\theta_{x, 2}^{e q \cdot}\right)
$$

Thus, the shear block strength along the beam-element is verified by making sure that:

$$
\frac{\left|T_{s}\right|}{T_{m} S} \leq 1
$$

where $S$ is the number of blocks per element and $T_{m}$ the shear block connection strength [23]. Accordingly, the strength verification for the shear block connections is performed at an element level rather than at single shear block connection level. 


\section{An optimisation method for variable cross-section}

Shell structures derive their capacity to resist inextensional deformations [24] from their inherent shape-resistant geometry. In fact, a (mainly) membrane stress field is formed as a response to external loading forces, thus allowing shells a with very low thickness/span ratio to be realized. Accordingly, the shape of a shell may be conceived as the result of an optimisation procedure, consisting of finding the funicular geometry for which the corresponding out-of-plane bending is null $[25,26,27,28]$. However, very small perturbations, such as support displacements, load or geometric imperfections, greatly reduce the theoretical load-carrying capacity of the funicular structure [9]. Therefore, even optimal (funicular) shapes require a 'certain amount' of bending stiffness for buckling and stability issues. Further, in current building design practice, the geometric definition of a shaperesistant structure (e.g. grid shell) is driven by a variety of design requirements, such as architectural, functional, thus resulting in shapes that differ widely from the funicular configuration [29]. Accordingly, additional stiffness may be required to enhance the structural capacity of such non-funicular shapes.

Designing the out-of-plane bending stiffness of a grid shell structure by 'adjusting' the thickness of its members, can be expected that for a given dominant load combination (e.g. dead load), the stiffness demand will vary among the members. Accordingly, a variable cross-sectional thickness can be sought for the entire system in order to meet the required load-carrying capacity while minimizing the amount of material. On this basis, a local search optimisation method for actively bent (double-layer) grid shells is introduced here. The method computes the optimal variable shear block's height $h_{s}$ such that the bending stress $\sigma$ at the external fibre of the cross-section is 'normalized' to a given $f_{m}$ value for the entire structure. More precisely, the cross-sectional thickness is proportionally scaled (at each step) according to the linearised field of bending stress ratios $\sigma / f_{m}$ resulting from a non-linear analysis (DR) with initially constant cross-section. The linearisation error decreases as the number of DR steps increases, up to a point for which, no substantial improvement is appreciated, thus the procedure is stopped.

Clearly, the lower the $f_{m}$ limit stress is assumed to be, the higher the bending stiffness will result from the optimisation process. As a consequence, for structural systems working mainly in bending action (the simply supported beam as 
'extreme' case), an $f_{m}$ value close to the material limit strength will be chosen. Conversely, for shape-resistant systems working mainly in compression, the choice of the 'uniforming' value $\left(f_{m}\right)$ will be mainly dictated by buckling.

\subsection{Single-rod system}

The method for cross-section optimization introduced here is suitable for the resolution of single-rod systems. Then, an updated procedure for grid shell frameworks, that takes into account additional geometric compatibility constrains, is illustrated in section 3.2.

Indicating the vector of design space $\mathbf{h}$ as:

$$
\mathbf{h}=\left\{h_{s, 1} \ldots h_{s, j} \ldots h_{s, n^{\circ}}\right\}
$$

with $h_{s, j}$ the shear block's thickness of the $j$ th beam-element, and $n^{\circ}$ the total number of elements of the system, the objective is to find the components of $\mathbf{h}$ that minimize the deviation of combined bending stresses (at the beam's external fibre) from the uniforming value $\left(f_{m}\right)$ and, at the same time, no stress ratio $\sigma / f_{m}$ is higher than unity. Indicating with $\Sigma$ the resultant of bending stress along the element's domain (from $t=0$ to $t=1$ ):

$$
\Sigma=\left|\Sigma_{x, a}\right|+\left|\Sigma_{x, b}\right|+\left|\Sigma_{y}\right|
$$

The constrained optimisation problem can be formally stated as:

$$
\text { minimize : } \quad f(\mathbf{h})=\sum_{j=1}^{n^{\circ}} \frac{1}{n^{\circ}}\left|\frac{\Sigma_{j}}{f_{m}}-1\right|
$$

315

$$
\text { subject to: }\left\{\begin{array}{l}
\Sigma_{j} \leq f_{m} \\
T_{s, j} \leq T_{m} S \\
h_{s, j} \geq 0
\end{array}\right.
$$

The stress terms in Eq. (42) are so defined:

$$
\begin{aligned}
\Sigma_{x, a} & =\int_{0}^{1} \sigma_{x, a}(t) d t=\frac{h E}{2|\bar{p}|}\left[\theta_{x, 1}-\theta_{x, 2}-\left(\theta_{x, 1}^{e q .}-\theta_{x, 2}^{e q \cdot}\right)\left(1-c_{R}\right)\right] \\
\Sigma_{x, b} & =\int_{0}^{1} \sigma_{x, b}(t) d t=\frac{E c_{s}\left(h+h_{s}\right)}{2|\bar{p}|}\left(\theta_{x, 1}-\theta_{x, 1}^{e q .}-\theta_{x, 2}+\theta_{x, 2}^{e q .}\right)
\end{aligned}
$$




$$
\Sigma_{y}=\int_{0}^{1} \sigma_{y}(t) d t=-\frac{b E}{2|\bar{p}|}\left[\theta_{y, 1}-\theta_{y, 2}-\left(\theta_{y, 1}^{e q .}-\theta_{y, 2}^{e q .}\right)\left(1-c_{R}\right)\right]
$$

Noting that: since $t$ is a dimensionless parameter, $\Sigma_{x, a}, \Sigma_{x, b}$ and $\Sigma_{y}$ in Eqs. (45

where:

$$
d_{j}=\max \left\{\begin{array}{l}
{\left[\frac{\left|\Sigma_{x, b}\right|\left(h+h_{s}\right)}{f_{m}-\left|\Sigma_{x, a}\right|-\left|\Sigma_{y}\right|}-h\right]_{j}} \\
\left(h_{s} \frac{\left|T_{s}\right|}{T_{m} S}\right)_{j} \\
0
\end{array}\right.
$$

A flowchart of the described method is shown in Figure 5.

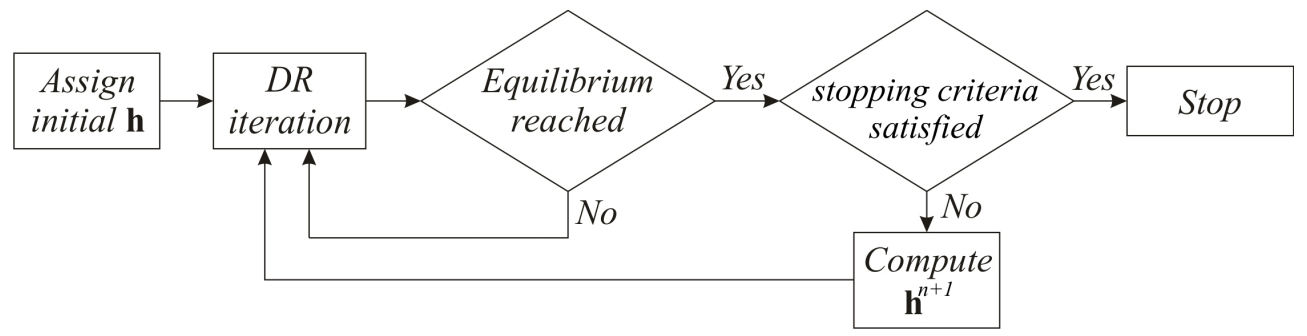

Figure 5: Flowchart of the method for cross-section optimisation.

\footnotetext{
${ }^{2}$ In fact: $\int_{0}^{1} \sigma(t) d t=\sigma(t=0.5)$
} 


\subsection{Two-way grid shells}

For three-dimensional systems, such as two-way grid shells (see Figure 1), constructional considerations mean that the thickness of the shear blocks needs to be

$$
h_{s, j} \geq h
$$

consequently, the zero in the third of Eq. (50) is replaced with $h$. Additionally, the thickness of the built-up cross-section of ribs in the two different directions need to be equal at the nodal intersection (see Figure $6 \mathrm{~b}$ ). Such geometric compatibility results in a further geometric constraint: Indicating with $i$ the generic node index and $u$ and $v$ the two-way grid directions, then the following equality constraint is added to Eqs. (44):

$$
h_{s, i}^{u}=h_{s, i}^{v}
$$

Noting that the superscripts $u$ and $v$ refer to the grid's directions (Figure $6 \mathrm{~b}$ ) while the superscript $n$ (e.g in Eqs. $(48$ - 49)) refers to the thickness value at the $n$th DR step. In order to solve the updated optimisation problem in an explicit way, the computing of $\mathbf{h}^{n+1}$ for a two-way grid shell system is performed (as before) according to Eqs. (49-50) but, in order to assure geometric compatibility imposed by Eq. (52), an additional operation is performed (at each DR step) on the $\mathbf{h}^{n+1}$ list resulting from Eq. (49).

(a)

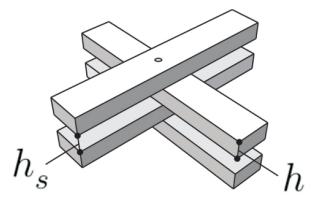

(b)

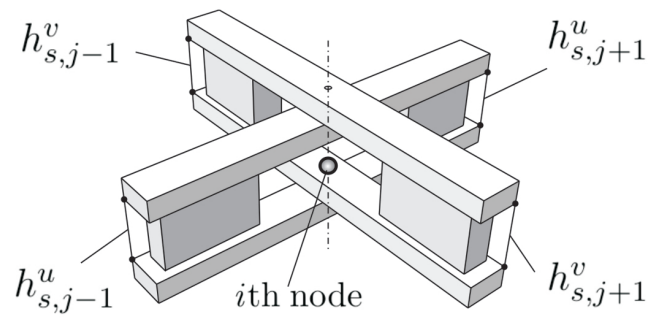

Figure 6: Geometric compatibility of thicknesses: (a) $h_{s} \geq h$; (b) Shear block thicknesses at the $i$ th node as average of the surrounding elements (see Eqs. (53)).

\subsubsection{Geometric compatibility}

The additional operation to perform on the $\mathbf{h}^{n+1}$ list (as from Eq. (49)) to assure geometric compatibility, is described as follows: Firstly, an average $h_{s, i}$ 
value, for the $i$ th node, is computed as a function of the shear blocks thickness of the surrounding elements belonging to the $u$ and $v$ directions. With reference to Figure 6b:

$$
h_{s, i}^{u}=\frac{h_{s, j-1}^{u}+h_{s, j+1}^{u}}{2} ; h_{s, i}^{v}=\frac{h_{s, j-1}^{v}+h_{s, j+1}^{v}}{2}
$$

Noting that the subscript $i$ refers to the thickness $h_{s}$ interpolated at the $i$ th node while the subscript $j$ refers to the (constant) thickness value $h_{s}$ of the $j$ th element. In other words, $h_{s}$ is assumed to vary 'linearly' along the element. Accordingly, for each $i$ node there will be two thickness values, each one interpolated according to the grid direction $(u, v)$. By taking, for the $i$ th node, the maximum value:

$$
h_{s, i}^{\max }=\max \left\{h_{s, i}^{u} ; h_{s, i}^{v}\right\}
$$

and writing the linear (thickness) variation law along the $j$ th element:

$$
\begin{aligned}
& h_{s}^{u}(t)=\left(h_{s, i_{2}}^{u}-h_{s, i_{1}}^{u}\right) t+h_{s, i_{1}}^{u} \quad ; \quad t \in[0,1] \\
& h_{s}^{v}(t)=\left(h_{s, i_{2}}^{v}-h_{s, i_{1}}^{v}\right) t+h_{s, i_{1}}^{v}
\end{aligned}
$$

the difference in values between $h_{s}$ as from the Eq. (49) and $h_{s}(t=0.5)$ as from Eqs. (55) is measured:

$$
\begin{aligned}
& h_{s}^{u}-\left[\left(h_{s, i_{2}}^{u}-h_{s, i_{1}}^{u}\right) 0.5+h_{s, i_{1}}^{u}\right] \\
& h_{s}^{v}-\left[\left(h_{s, i_{2}}^{v}-h_{s, i_{1}}^{v}\right) 0.5+h_{s, i_{1}}^{v}\right]
\end{aligned}
$$

thus: the linear thickness variation law is updated this time by considering, for each element's end, the maximum value $h_{s, i}^{\max }$ as from Eq. (54). Accordingly, Eqs. (55) become:

$$
\begin{aligned}
& h_{s}^{u}(t)=\left(h_{s, i_{2}}^{\max }-h_{s, i_{1}}^{\max }\right) t+h_{s, i_{1}}^{\max } \\
& h_{s}^{v}(t)=\left(h_{s, i_{2}}^{\max }-h_{s, i_{1}}^{\max }\right) t+h_{s, i_{1}}^{\max }
\end{aligned}
$$

The new 'constant' $h_{s}^{*}$ value for the $j$ th element is obtained by setting $t=0.5$ in Eqs. (57) and adding them up to the quantities in (56) hence, obtaining the following general equation:

$$
h_{s}^{*}=h_{s}+\frac{1}{2}\left(h_{s, i_{2}}^{\max }-h_{s, i_{1}}^{\max }-h_{s, i_{2}}+h_{s, i_{1}}\right)+h_{s, i_{1}}^{\max }-h_{s, i_{1}}
$$

in which $h_{s}$ is that obtained by Eq. (49). As it can be seen: for $h_{s, i_{1}}=h_{s, i_{1}}^{\max }$ and $h_{s, i_{2}}=h_{s, i_{2}}^{\max } \Rightarrow h_{s}^{*}$ and $h_{s}$ are equal, therefore, the consistency between 'constant' and 'linear' models, of thickness along the element, is held. 
It is important here to note that: an average value between $h_{s, i}^{u}$ and $h_{s, i}^{v}$ in replacement of $h_{s, i}^{\max }$ in Eq. (54), would allow for a greater minimization of the objective function as stated in Eq. (43). Nevertheless, by considering $h_{s, i}^{\max }$ makes it possible to fulfil the strength (optimisation) constraint stated in the first of Eqs. (44) according to which, no bending stress ratios higher than unity must occur. For single-rod systems (Subsection 3.1) such strength constraint is 'implicitly' fulfilled by Eqs. (50).

The accuracy of the co-rotational finite element theory, is tested by comparison with analytical and experimental results (Section 4) while Section 5 reports two examples on the application of the optimization method described above.

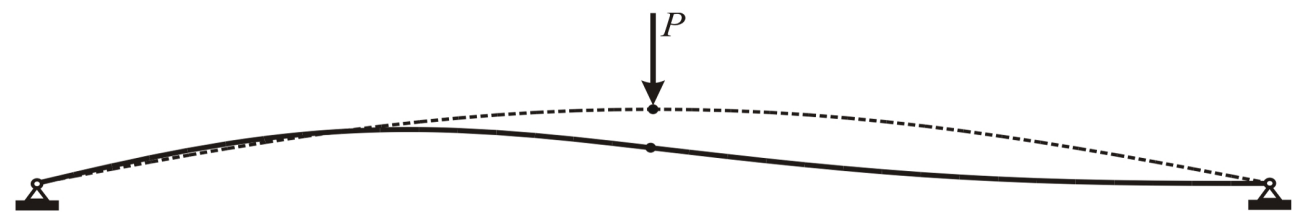

Figure 7: Shallow arch subjected to a nodal load $P$ applied at the mid-span: The bold line represents the asymmetric buckled shape.

\section{Preliminary Calculations}

\subsection{Elastic buckling of shallow arches}

The described modified co-rotational formulation is firstly tested by computing the elastic buckling loads of a shallow arch subjected to an applied nodal load $P$ at the mid-span (see Figure 7). The arch geometry is obtained by pre-bending a straight elastic rod. The rod's length $(L)$ is $320 \mathrm{~mm}$, with an axial stiffness $E A=5 \mathrm{MN}$ and bending stiffness $E I=10 \mathrm{Nm}^{2}$. A preliminary DR analysis was performed to generate the pre-bent configuration, thus obtaining an arch with rise $H=20.6 \mathrm{~mm}$ and a span of circa $316.5 \mathrm{~mm}$.

Two sets of analyses are carried out:

- Pre-stressed configuration: $\left(c_{R}=1\right)$.

- Stress-free configuration: $\left(c_{R}=0\right)$.

For each set of analyses, a displacement controlled technique is adopted, by imposing a vertical displacement increment of $0.1 \mathrm{~mm}$ to the mid-span node, and 
allowing the DR routine to reach the corresponding equilibrium configuration, after which, the corresponding vertical reaction force at the midspan node $(R=-P)$ is recorded and a further displacement increment is set.

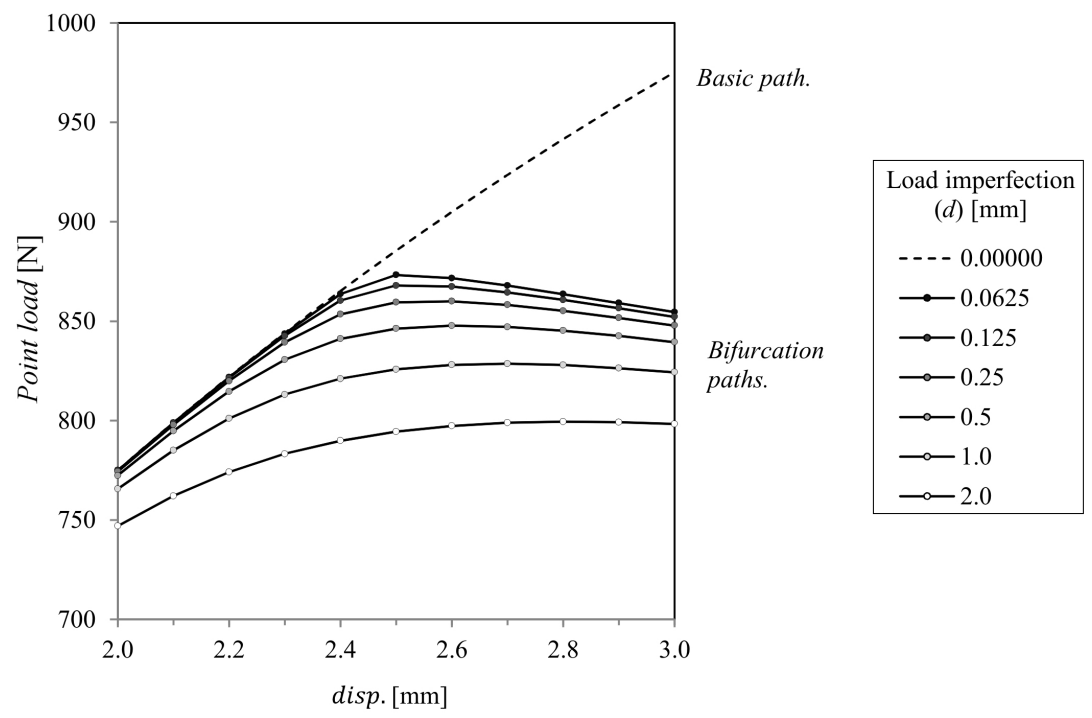

Figure 8: Bifurcation paths for different imperfection's amplitudes of the pre-stressed arch $\left(c_{R}=1\right)$.

The DR stopping criteria for equilibrium convergence (for all the analyses described in this paper) was set to: $\max \left|\bar{R}_{i}\right| \leq 0.001 \mathrm{~N}$, with $\bar{R}_{i}$ the out-of-balance force at the $i$ th node. Further, for each set of analyses, a load imperfection is introduced by offsetting the point load a certain distance $(d)$ towards the right of the arch centreline. Such load imperfection is numerically simulated by applying the imposed vertical displacement at the centreline node but adding an applied torque to it as well. The torque's magnitude is set according to the length of the lever arm $(d)$ and updated at each DR increment of time $(t+\Delta t)$ as a function of the reaction force $(R=-P)$ recorded at time $t$.

The bifurcation paths for different amplitudes of imperfection of the pre-stressed configuration $\left(c_{R}=1\right)$ are reported in Figure 8. Accordingly, for each analysis, the maximum recorded $P$ value is assumed to be the elastic buckling load value as reported in Figure 9, where a comparison with the corresponding analytical solution is made. The analytical elastic buckling load of pre-stressed and stress-free shallow 


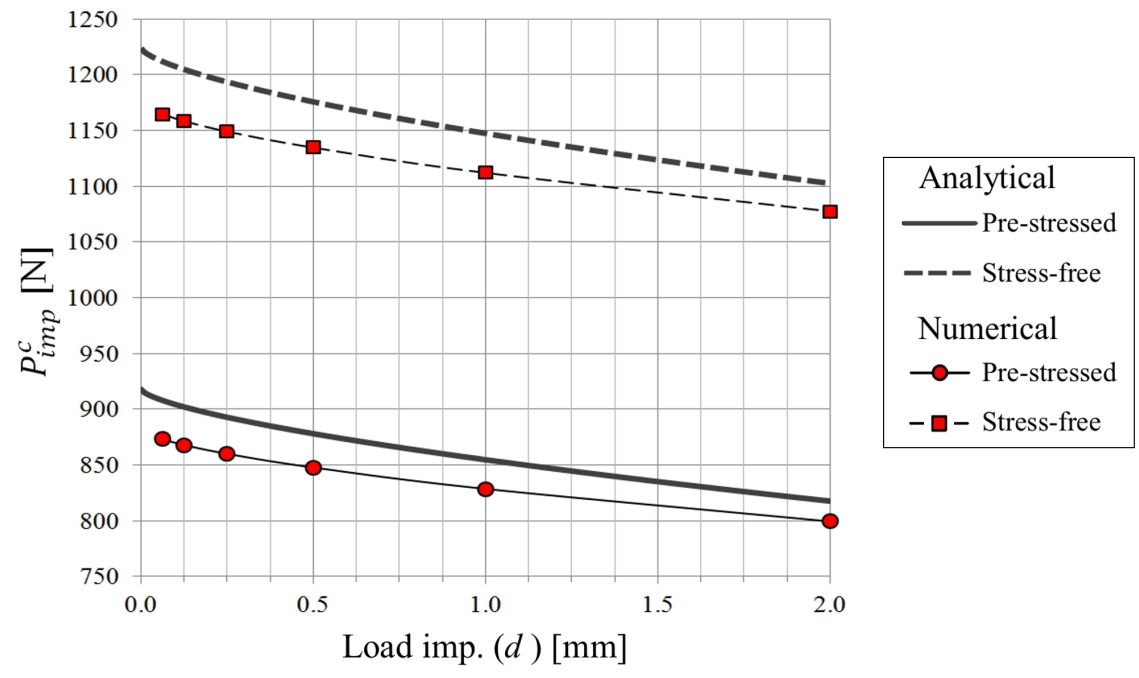

Figure 9: Comparisons of analytical and numerical buckling loads for different imperfection's amplitudes of the pre-stressed and stress-free arch.

arches is given by [30]:

$$
P_{i m p .}^{c}=\left(\frac{3}{2} \frac{\pi^{4} E I H}{L^{3}}\right)\left[1-3.22\left(\frac{d}{L}\right)^{\frac{2}{3}}\right]
$$

415

Stress-free arch

$$
P_{i m p .}^{c}=\left(2 \frac{\pi^{4} E I H}{L^{3}}\right)\left[1-2.92\left(\frac{d}{L}\right)^{\frac{2}{3}}\right]
$$

As expected, the numerical buckling load decreases, together with the analytical load, as the imperfection $d$ is increased (see Figure 9). The numerical values are consistently lower than the analytical values, with a maximum deviation, for $d=$ $0.0625 \mathrm{~mm}$, of circa $-3.8 \%(-47.5 \mathrm{~N})$ for the stress-free arch (and an absolute vertical displacement of $3.1 \mathrm{~mm}$ at the buckling point). A max. deviation of $-3.8 \%$ ($34.9 \mathrm{~N}$ ) is found for the pre-stressed arch as well, with a vertical displacement of $2.5 \mathrm{~mm}$ at the buckling point. Such discrepancy may be due to the inextentional theory adopted for the derivation of the analytical formulae [30] leading to buckling load values of shallow arches with infinite axial stiffness. At the buckling point 
(for $d=0.0625 \mathrm{~mm}$ ) both pre-stressed and stress-free arches were around $0.076 \%$ shorter $(-0.24 \mathrm{~mm})$. Such (apparently negligible) shortening is consistent with the discrepancy between numerical and analytical buckling loads. In fact, according to Timoshenko and Gere [31], the influence of axial deformation greatly reduces the buckling load, as the rise/span ratio of the arch is reduced as well.

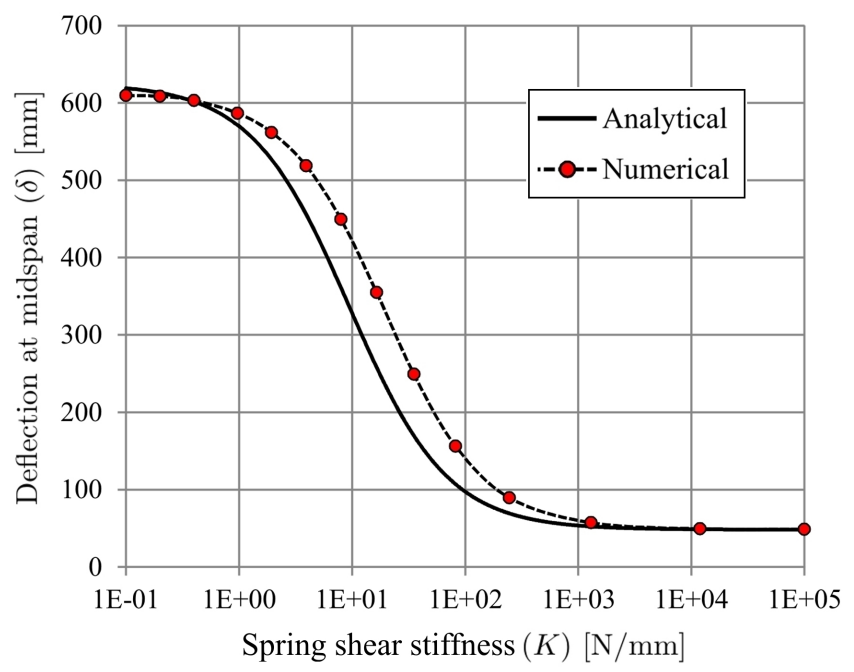

Figure 10: Simply supported beam: comparison of numerical and analytical outputs of deflection at the midspan as a function of the spring (fastener) stiffness $(K)$.

\subsection{Double-layer simply supported beam}

In the following example, a double-layer simply supported beam is loaded with a uniformly distributed load $W$ of $50 \mathrm{~N} / \mathrm{m}$. The beam's length $(L)$ is $10 \mathrm{~m}$ and an elastic modulus $E$ of $10 \mathrm{kN} / \mathrm{mm}^{2}$ is chosen, while a cross-section with $b=h=h_{s}=$ $50 \mathrm{~mm}$ is set. Further, a spring (fastener) spacing $s=100 \mathrm{~mm}$ is considered (see Eq. (19)). On this basis, the beam's deflection at the midspan $(\delta)$ is analytically computed according to the following equation [3]:

$$
\delta=\frac{W L^{4}}{\frac{26}{12} b^{4} E}\left\{\frac{5}{384}+\frac{3}{8 \theta}\left[1+\frac{2}{\theta}\left(\frac{1}{\cosh \sqrt{\theta}}-1\right)\right]\right\} \quad ; \quad \theta=\frac{26 K L^{2}}{4 s b^{2} E}
$$

for different values of the shear stiffness of the springs $(K)$ varying from 0.1 to $10^{5} \mathrm{~N} / \mathrm{mm}$. In order to numerically compute the beam's deflection, the connection efficiency factor is derived by applying the EC5 Eq. (20) according to which: 
for $K=0.1 \Rightarrow c_{s}=0.0004$ while for $K=10^{5} \Rightarrow c_{s}=0.997$. The analytical and numerical outputs of the midspan deflection are compared in Figure 10 as a function of the spring stiffness (reported on a logarithmic scale with base 10): As can be seen, for $1<K<1000\left(0.0039<c_{s}<0.8\right)$ the numerical model shows a much lower bending stiffness compared to the analytical one. This is due to the EC5 function in Eq. (20), which provides conservative values for the connection efficiency factor $c_{s}$.

(a)

(b)
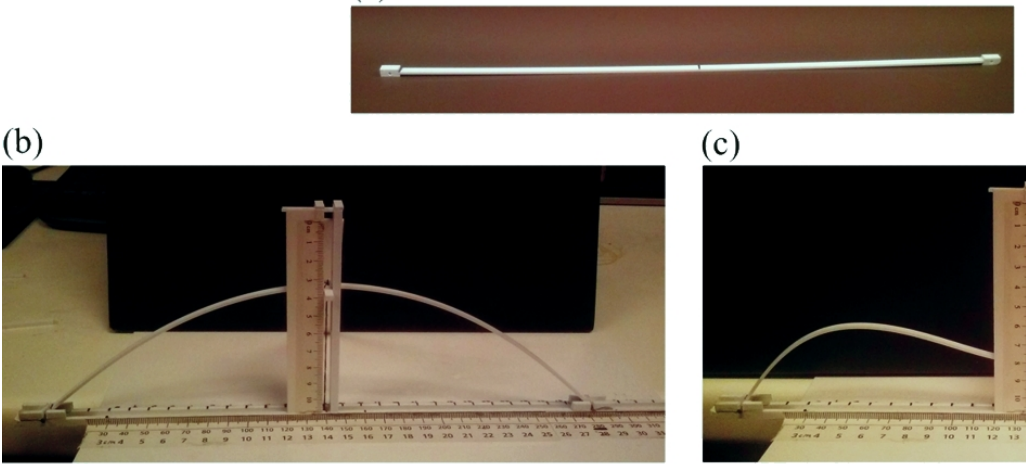

(c)

(d)

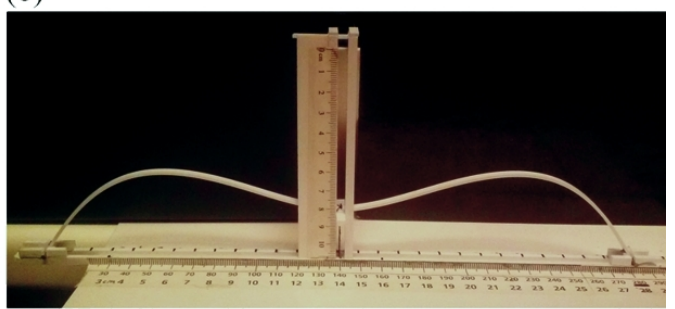

(e)
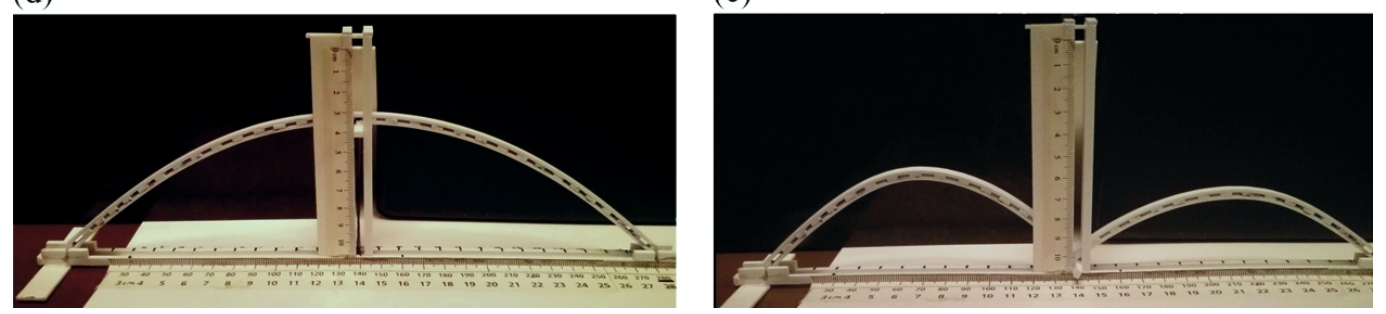

Figure 11: Experimental test: (a) Styrene lath; (b) Pre-stressed single-layer arch; (c) Symmetric buckling of the pre-stressed single-layer arch; (d) Pre-stressed double-layer arch; (e) Failure of pre-stressed double-layer arch.

\subsection{Double-layer pre-stressed arch}

To further assess the effectiveness of the the modified co-rotational formulation for double-layer members (assuming an equivalent $E I$ ), the load-deflection curves of a single and double-layer pre-stressed arch, subjected to point load $P$ at the midspan, are evaluated by experimental physical test and compared to the corresponding numerical values. The initially straight lath (see Figure 11) is made from Styrene (polymer) with a length $L=320 \mathrm{~mm}$, cross-section $b \times h=$ 
$3.97 \mathrm{~mm} \times 1.48 \mathrm{~mm}$ and Young's modulus $E=2140 \mathrm{~N} / \mathrm{mm}^{2}$, which was assessed by a preliminary tension test carried out according to EN ISO 527-1 [32]. The resulting arches have a span of $280 \mathrm{~mm}$. Pinned restrains are provided at ends of the arches, thus allowing only rotational degrees of freedom.

\subsubsection{Experimental set-up}

A first physical test is carried out on the single Elastica arch (Figures $11 \mathrm{~b}$ and 11c) thus, assuming the recorded experimental load $P$ as halved the load that would result from a double-layer pre-stressed arch without shear blocks. A second test is then conducted on the double-layer arch (Figures 11d; 11e) in which, the built-up cross-section is obtained by welding (with Dichloromethane) 32 evenly spaced shear blocks $\left(h_{s}=h=1.48 \mathrm{~mm}\right)$ on the pre-bent single arch, then, with the lower (pre-bent) lath in place, the upper lath is bent and welded on the shear blocks as well. Since the end restraints are aligned with the cross-section of the lower lath, the resulting experimental set-up does not perfectly match with the numerical model (where the end restraints are aligned with the barycenter of the double-layer cross-section). Nevertheless, the described set-up faithfully resembles the construction method of a real (actively bent) arch.

Lateral and asymmetric buckling are prevented by positioning vertical supports sideways, along the arch centreline, therefore, the displacements are recorded on a metric scale positioned perpendicular to the arch. The load is incrementally applied by placing nails (weighing $2.18 \mathrm{~g}$ each) into a basket hanging underneath the structure and tied to the arch midspan by means of wire.

\subsubsection{Comparison of results}

Unlike physical tests, a displacement controlled technique (displacement increment $=2.5 \mathrm{~mm}$ ) was adopted to numerically trace the load-displacement curves of the double-layer arches over the buckling point. In order to assess the effect of pre-stress forces, two analyses were performed for each shear block configuration, a total of four numerical analyses:

- $K=0\left(c_{s}=0\right) ;$ Stress-free $\operatorname{arch}\left(c_{R}=0\right)$.

- $K=0\left(c_{s}=0\right)$; Pre-stressed $\operatorname{arch}\left(c_{R}=1\right)$.

- $K=\infty\left(c_{s}=1\right) ;$ Stress-free $\operatorname{arch}\left(c_{R}=0\right)$. 
- $K=\infty\left(c_{s}=1\right)$; Pre-stressed $\operatorname{arch}\left(c_{R}=1\right)$.

For a better comparison of results, both experimental and numerical curves have been normalized according to the corresponding (numerical) elastic buckling loads $P^{c}\left(c_{R}=0\right)$ and reported in Figure 12.

(a)

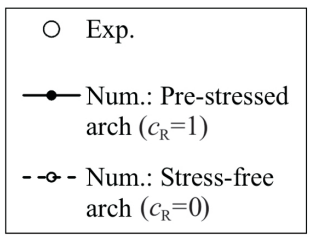

(b)
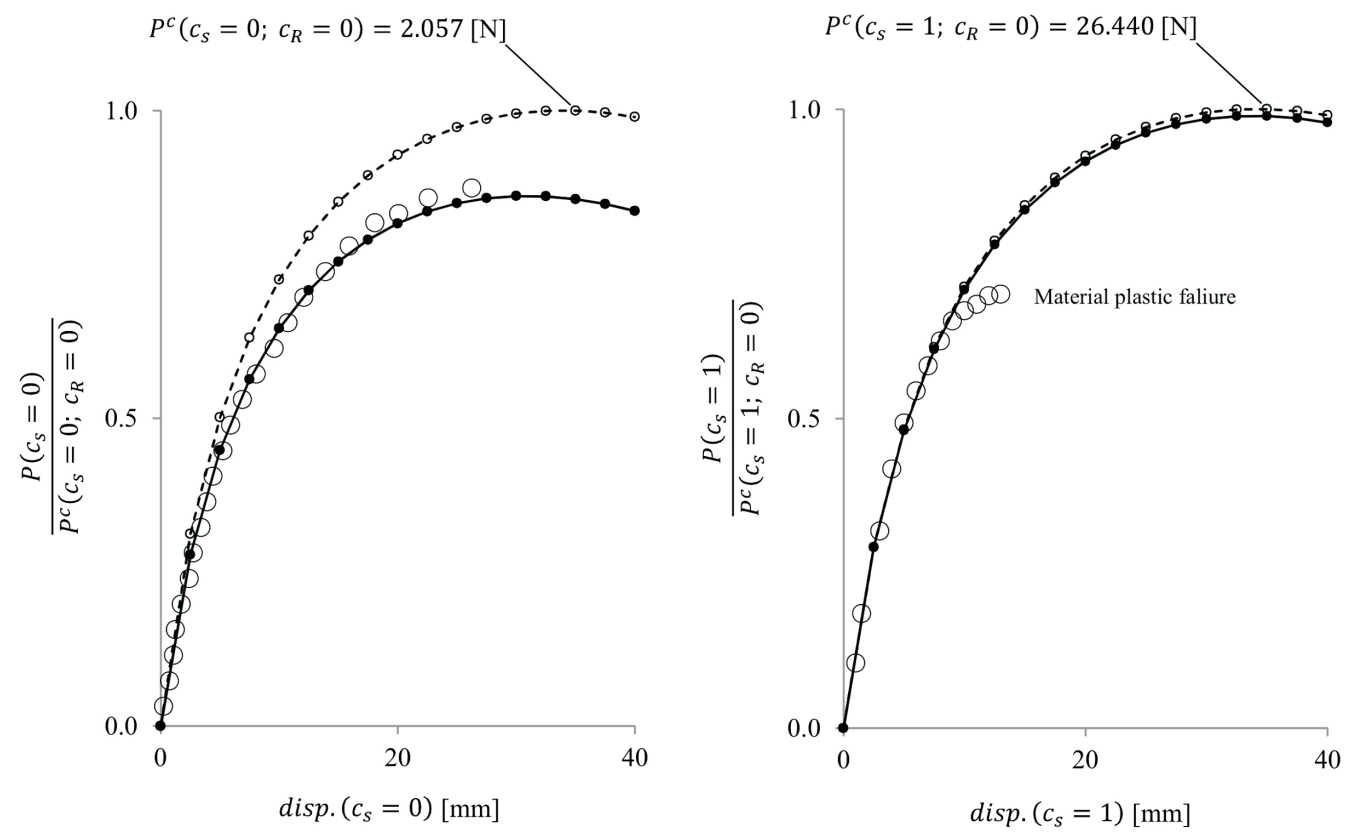

Figure 12: Comparison of experimental and numerical load-displacement curves: (a) Shear block connection stiffness $K=0\left(c_{s}=0\right)$; (b) Shear block connection stiffness $K=\infty\left(c_{s}=1\right)$.

As can be seen, the numerical curves are in good agreement with those obtained experimentally, with the exception of the last piece of experimental curve for the double layer arch (see Figure 12b) where, due to plastic failure of styrene (Figure 11e) the experimental load does not reach the elastic buckling value. As expected, the effect of shear blocks massively increases the buckling load from $2.057 \mathrm{~N}$ to $26.440 \mathrm{~N}$ (over 1100\%). Interestingly, in Figure 12a the negative effect of residual pre-stress on the buckling load is shown, which reduces of circa $14 \%$, thus in 
agreement with previous investigations [33]. Nevertheless, as already pointed out [34], Figure 12b shows that the lowering effect of pre-stress forces on the buckling load of double-layer arches, reduces with the increase of the shear blocks stiffness $K$, up to $K=\infty\left(c_{s}=1\right)$ at which point, pre-stress forces have a negligible effect (the buckling load reduction is only $1.12 \%$ ).

\section{Variable cross-section optimisation: Calculation}

The theory introduced in Section 3 for an iterative optimisation of the doublelayer cross-section is applied to the resolution of a simply supported beam and grid-shell structure.

(a)

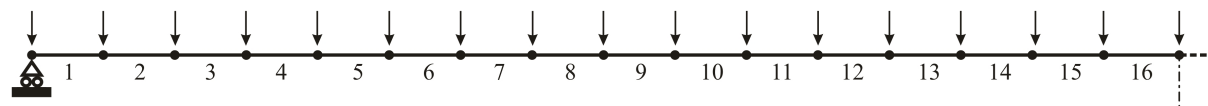

(b)

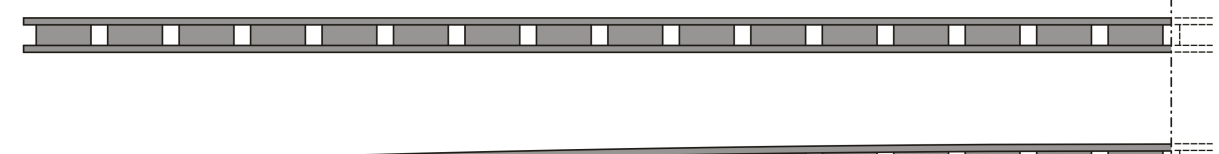

(c)

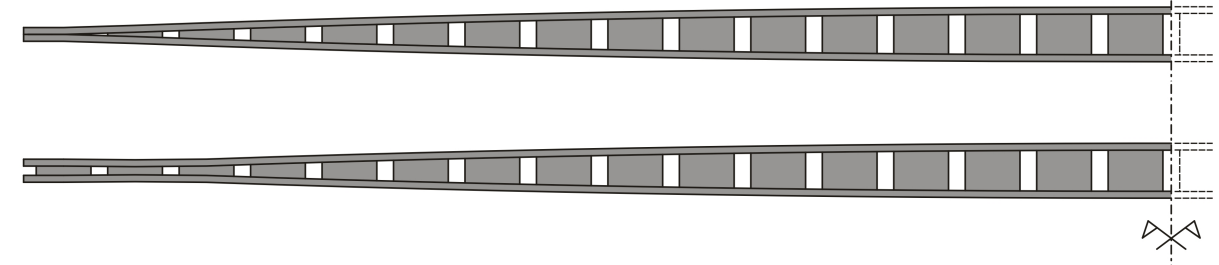

Figure 13: Simply supported beam: (a) Static scheme; (b) Initial cross-section; (c) Optimized cross-section for $T_{m} S=40 \mathrm{kN}$; (d) Optimized cross-section for $T_{m} S=20 \mathrm{kN}$.

\subsection{Simply supported beam}

A $10 \mathrm{~m}$ long beam, subjected to a uniformly distributed load of $1 \mathrm{kN} / \mathrm{m}$, is selected for the first analysis test. The static scheme of the system is shown in Figure 13a. The rectangular cross-section of the single lath is $b=80 \mathrm{~mm}$ and $h=30 \mathrm{~mm}$, while a constant shear blocks thickness $h_{s}=90 \mathrm{~mm}$ is initially assumed. The elastic modulus $E$ is set to $10 \mathrm{kN} / \mathrm{mm}^{2}$.

Since the numerical investigation is conducted on a straight beam, the value of $c_{R}$ does not affect the solution and is thus neglected, while, a connection efficiency factor $c_{s}=1$ and a stress limit value $f_{m}=28 \mathrm{~N} / \mathrm{mm}^{2}$ are assumed. The beam is discretised into 32 elements of uniform length. 


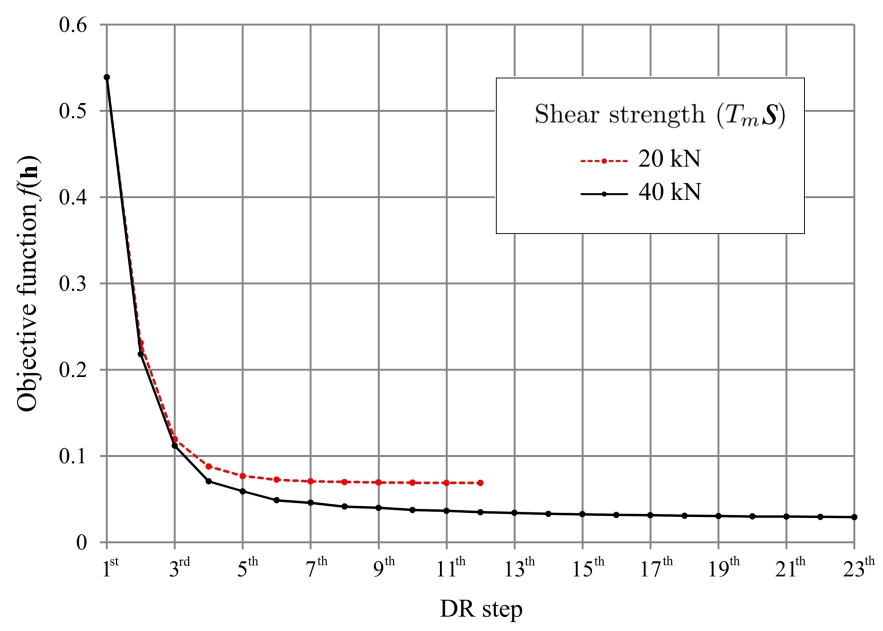

Figure 14: Simply supported beam: Optimisation's history for two different values of the strength constrain $T_{m} S$. The objective function $f(\mathbf{h})$ is computed according to Eq. (43).

The optimisation method was run twice, assuming shear strength limits $T_{m} S=$ $40 \mathrm{kN}$ and $20 \mathrm{kN}$ respectively (see Figure 14). Both analyses were stopped when: $\max \left\{\left|h_{s, j}^{n+1}-h_{s, j}^{n}\right|\right\} \leq 2 \mathrm{~mm}$. The initial and optimized beam profiles obtained with $T_{m} S=40 \mathrm{kN}$ and $T_{m} S=20 \mathrm{kN}$ are shown in Figures 13c and 13d respectively, while in Table 1 the stress ratio $\Sigma / f_{m}$ and the shear reaction $T_{s}$ are reported according to the element index (as reported in Figure 13a).

As can be seen in the third and fourth columns of Table 1, the bending stress ratios of the optimised beam are all unitary, with the exception of those in proximity of the beam supports as a consequence of the geometric and shear strength constraints (second and third of Eq. (44) respectively). As one would expect, in the fifth column of the same table, it can be seen that the beam with constant crosssectional height experiences a maximum horizontal shear reaction at the supports (left node of element 1) which decreases linearly up to zero at the beam midspan (right node of element 16). It is worth noting also that the reduced shear strength parameter $T_{m} S=20 \mathrm{kN}$, used to generate the values in the seventh column of Table 1, resulted in a beam with thicker cross-section at its end supports, as shown in Figure 13d in comparison to the one obtained by $T_{m} S=40 \mathrm{kN}$ (Figure 13c). 
Table 1: Simply supported beam: Comparisons, between the initial and optimized cross-section, in terms of bending stress ratios and shear reactions.

\begin{tabular}{lcccccc}
\hline Cross-section: & Constant & \multicolumn{2}{c}{ Optimized } & Constant & \multicolumn{2}{c}{ Optimized } \\
\hline$f_{m}\left[\mathrm{~N} / \mathrm{mm}^{2}\right]$ & 28 & 28 & 28 & & 28 & 28 \\
$T_{m} S[\mathrm{kN}]$ & & 40 & 20 & & 40 & 20 \\
\hline Elem. index ${ }^{\mathrm{a}}$ & \multicolumn{2}{c}{ Bending stress ratio $\left(\Sigma / f_{m}\right)$} & \multicolumn{2}{c}{ Shear reaction $\left(T_{s}[\mathrm{kN}]\right)$} \\
\cline { 2 - 7 } 1 & 0.120 & 0.562 & 0.218 & 12.0 & 37.4 & 20.0 \\
2 & 0.336 & 0.991 & 0.685 & 11.3 & 25.1 & 20.0 \\
3 & 0.538 & 0.999 & 0.998 & 10.5 & 16.9 & 17.3 \\
4 & 0.725 & 0.999 & 1.001 & 9.7 & 12.7 & 12.7 \\
5 & 0.898 & 0.999 & 1.000 & 9.0 & 9.9 & 9.9 \\
6 & 1.058 & 0.999 & 1.000 & 8.2 & 7.9 & 7.9 \\
7 & 1.202 & 0.999 & 1.000 & 7.4 & 6.4 & 6.4 \\
8 & 1.333 & 1.000 & 1.000 & 6.7 & 5.2 & 5.2 \\
9 & 1.450 & 1.000 & 1.000 & 5.9 & 4.3 & 4.3 \\
10 & 1.552 & 1.000 & 1.000 & 5.1 & 3.5 & 3.5 \\
11 & 1.640 & 1.000 & 1.000 & 4.4 & 2.8 & 2.8 \\
12 & 1.713 & 1.000 & 1.000 & 3.6 & 2.2 & 2.2 \\
13 & 1.771 & 1.000 & 1.000 & 2.8 & 1.7 & 1.7 \\
14 & 1.816 & 1.000 & 1.000 & 2.0 & 1.2 & 1.2 \\
15 & 1.845 & 1.000 & 1.000 & 1.2 & 0.7 & 0.7 \\
16 & 1.860 & 1.000 & 1.000 & 0.4 & 0.2 & 0.2 \\
\hline
\end{tabular}

${ }^{\mathrm{a}}$ as shown in Figure 13.

\subsection{Grid shell system}

The optimisation method is further tested on a simple grid shell system obtained by the preliminary bending of a flat mat geometry as shown in Figure 15. The initial two-way mat is made out of straight elastic rods evenly spaced at a distance of $2 \mathrm{~m}$. Then, an anti-gravitational load is applied at the central nodes, while the boundary nodes at the mat's corners are constrained to translate in the horizontal plane. Then, in a second preliminary step, the rollers at the supports are substituted by pinned restraints and the anti-gravitational load is removed, thus allowing the post formed grid shell to settle in its final configuration. The resulting geometry has a size of $18.7 \mathrm{~m} \times 18.7 \mathrm{~m}$ in plan and an elevation of $4.25 \mathrm{~m}$ (see Figure $15 \mathrm{~b}$ ).

On the equilibrium geometry so found, a connection efficiency factor $c_{s}=1$ and a $c_{R}$ factor $=0$ (Stress-free geometry) are set.

A gravitational load of $2 \mathrm{kN}$ per node is considered for the optimization process. 
(a)

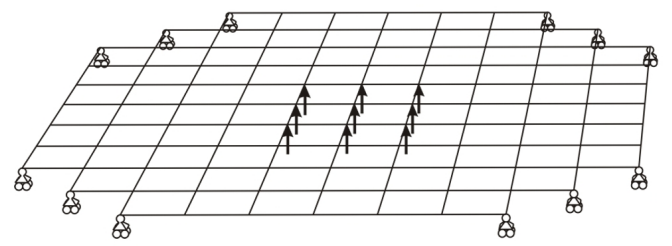

(b)

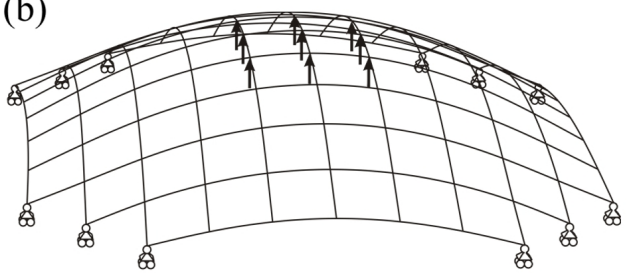

Figure 15: Grid shell System: (a) Initial flat mat; (b) post formed shape.

The initial cross-sectional geometry (with constant $h_{s}$ ) is set in accordance with the test described in Section 5.1 (see Figure 17a) as well as the Young's modulus. In addition, a shear modulus $G=700 \mathrm{~N} / \mathrm{mm}^{2}$ and a $f_{m}$ value of $8 \mathrm{~N} / \mathrm{mm}^{2}$ are considered. As for the simply supported beam, the convergence criterion was set according to: $\max \left\{\left|h_{s, j}^{n+1}-h_{s, j}^{n}\right|\right\} \leq 2 \mathrm{~mm}$.

As can be seen from Figure 16, after five DR steps, the average stress ratio $f(\mathbf{h})$ of Eq. (43) does not experience any minimization but maintains a steady value of circa 0.5. Nonetheless, the maximum bending stress ratio $\Sigma / f_{m}$ converges to unity, dropping from 1.8 (at completion of the first DR step) to 1.03 (at completion of the fifth DR step) therefore fulfilling the optimisation constraint stated in the first of Eqs. (44). The optimized geometry is shown in Figure 17b.

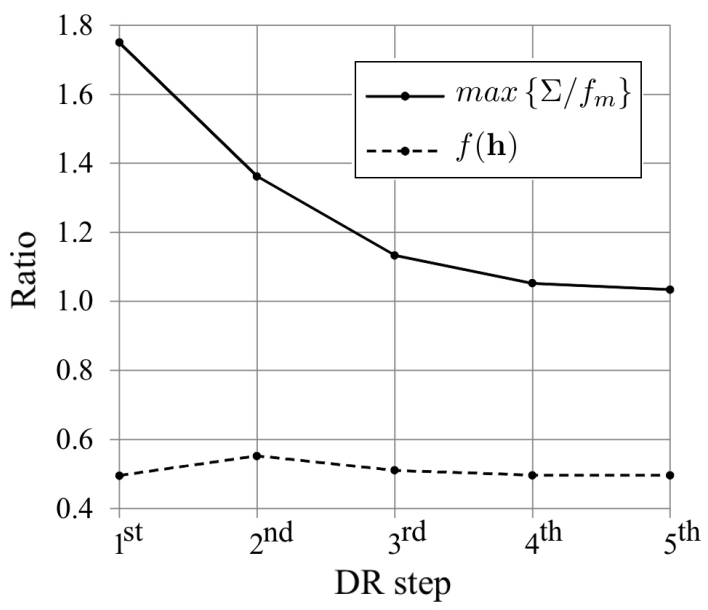

Figure 16: Grid shell System: Optimisation history in terms of maximum bending stress ratio $\Sigma / f_{m}$ and average stress ratio $f(\mathbf{h})$ as expressed in Eq. (43). 

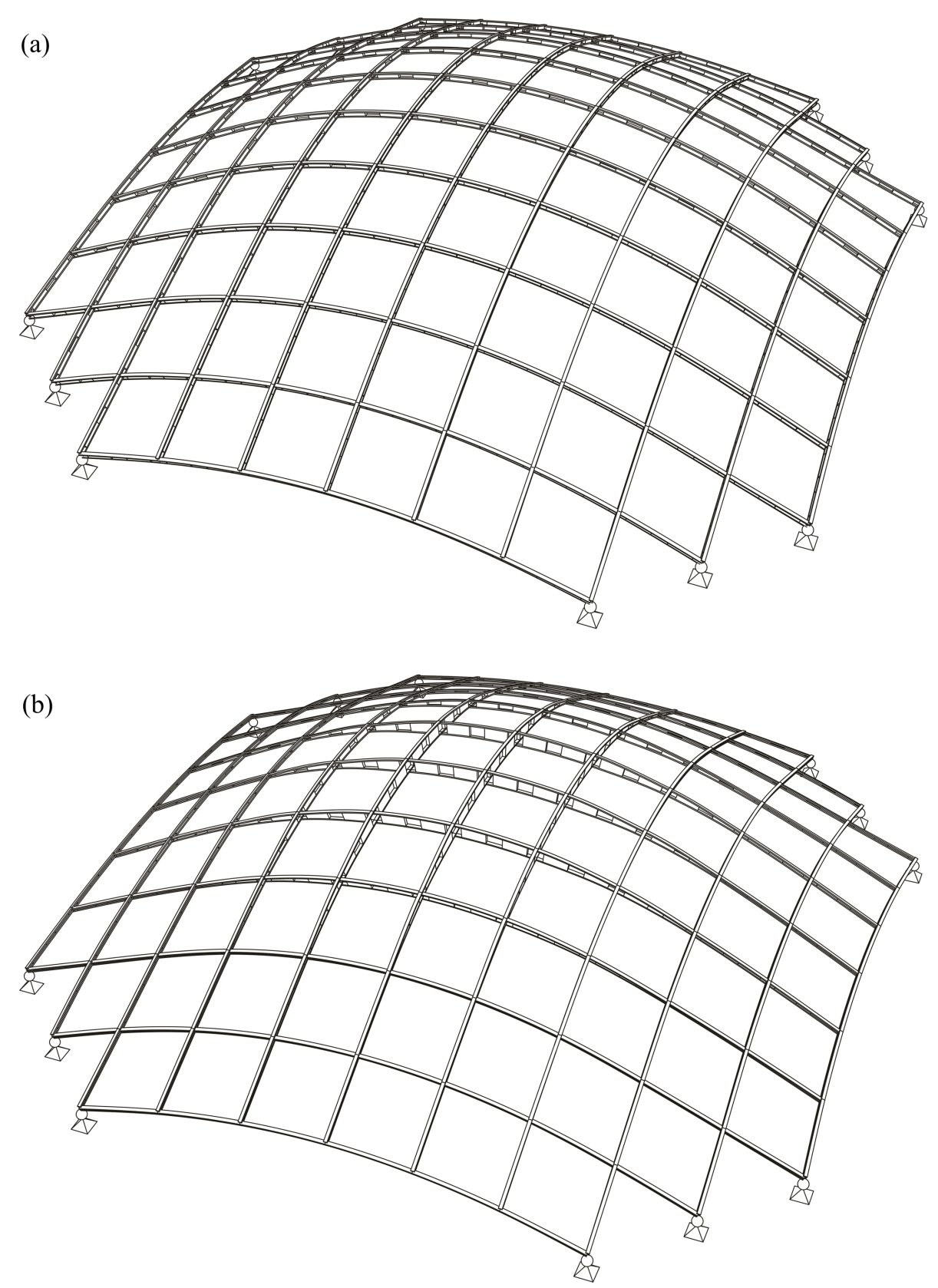

Figure 17: Grid shell System: (a) Constant cross-section $\left(h_{s}=90 \mathrm{~mm}\right)$; (b) Optimized crosssection. 


\section{Conclusions}

In this paper, a method for optimisation of the cross-section of actively bent structural systems is introduced. For a given load distribution, an optimal thickness variation of the built-up members (represented by the vector of design space $\mathbf{h}$ ) is sought in order to homogenize the bending stress to a given value $f_{m}$, thus allowing for optimal material distribution. Strength limit constraints are taken into account in the optimisation problem, as well as 'constructional' constraints, which are expressed in terms of geometric compatibility.

To model the effect of residual forces (due to the forming process) and change in stiffness of actively bent members, a modified co-rotational beam-element formulation for handling large displacements, is developed and checked against the analytical solutions of standard test cases, such as a simply supported beam and a shallow arch, as well as small scale physical tests. The modified co-rotational formulation is based upon assumption of an 'equivalent' $E I$ for the modelling of the built-up members. As pointed out in Subsection 2.2, such a 'simplified' approach does not takes into account the effect of shear flexibility, thus its applications should be limited to relatively thin members.

In addition to the construction (bending) process simulation and structural analysis, the design of actively bent structures provides a preliminary form finding stage, in which the initial configuration may be far enough from equilibrium to be practically intractable with implicit Finite Element schemes using NewtonRaphson solver (lack of numerical convergence). Accordingly, an explicit resolution method (DR) is here adopted in conjunction with the Finite Element co-rotational formulation. The material formulation was limited in here to the linear-elastic case, nevertheless, it can be easily extended to take into account material's nonlinearities.

Although focus was only given in here on two-way grid shells with constant member's length, the described formulations can be applied to any kind of actively bent structural system involving the use of initially straight or naturally curved beams/rods, as for instance, geodesic rib shells [37, 38], interlaced space structures [39] or hybrid systems, such as tension structures with integrated actively bent elements [40].

In section 5, the method's efficiency in finding an optimal distribution of the 
member's thickness is demonstrated on a single-rod structural system as well as a

grid shell framework, for which, a linear variation law $h_{s}(t)$ is adopted (see Eqs. $(55,57))$ to allow the inclusion of constructional (geometric) constrains into the optimization problem. As previously mentioned in the introduction, such linear function, describing the thickness variation along the element, can be used to inform the fabrication process by providing a mass-customized 'trapezoidal' profile $\left( \pm h_{s}(t) / 2\right)$ for the shear blocks, to be fabricated e.g by CNC cutting machinery, thus allowing minimization of 'gaps' at the connection interface between shear block and upper/lower lath. The gap's reduction at the interfaces, rises the potentials for realization of glued connections for the shear blocks, therefore providing a 'massive' increment of the out-of-plane bending stiffness (as shown in section 4.3.2) as well as the vanishing of the lowering effect (due to pre-stress forces) on the elastic buckling load.

\section{Acknowledgements}

The Centre for Timber Engineering (CTE) and the School of Engineering and Built Environment (SEBE) at Edinburgh Napier University are gratefully acknowledged for providing the financial support for this research. Thanks are also extended to Dr Callum Wilson for carrying preliminary tension tests on the Styrene laths used for the experimental test described in section 4.3.

\section{References}

[1] J. Lienhard, H. Alpermann, C. Gengnagel, J. Knippers, Active bending, a review on structures where bending is used as a self-formation process, International Journal of Space Structures 28 (3) (2013) 187-196. doi:http: //dx.doi.org/10.1260/0266-3511.28.3-4.187.

[2] C. Douthe, O. Baverel, J.-F. Caron, Form-finding of a grid shell in composite materials, Journal-International association for shell and Spatial Structures 150 (2006) 53.

[3] E. Happold, W. Liddell, Timber lattice roof for the mannheim bundesgartenschau, The structural engineer 53 (3) (1975) 99-135. 
[4] R. Harris, J. Romer, O. Kelly, S. Johnson, Design and construction of the downland gridshell, Building Research \& Information 31 (6) (2003) 427-454. doi:http://dx.doi.org/10.1080/0961321032000088007.

[5] R. Harris, S. Haskins, J. Roynon, The savill garden gridshell: design and construction, The Structural Engineer 86 (17) (2008) 27-34.

[6] S. Pone, B. D'Amico, S. Colabella, B. Parenti, D. Lancia, A. Fiore et al, Construction and form-finding of a post-formed timber grid-shell, in: Structures and architecture. Concepts, Applications and challenges, 2013, pp. 245-252. doi:http://dx.doi.org/10.1201/b15267-32.

[7] S. Pone, S. Colabella, B. D'Amico, D. Lancia, A. Fiore, B. Parenti, Timber post-formed gridshell: Digital form-finding / drawing and building tool, in: Proc. of the IASS Symposium, Wroclaw, Poland, 2013. doi:http://dx.doi . org/10.13140/2.1.1339.1686.

[8] B. DAmico, A. Kermani, H. Zhang, A form finding tool for post formed timber grid shells, in: proceedings of the World Conference on Timber Engineering, Quebec City, Canada, 2014. doi:http://dx.doi.org/10.13140/2.1.3949. 8888.

[9] Shell Structures for Architecture, Form finding and Optimization, Taylor and Francis, 2014.

[10] R. Harris, The timber gridshell (power-point slides), Presented at the COST Action Training School 2012, Lund University, Lund, Sweden.

[11] T. Belytschko, L. W. Glaum, Applications of higher order corotational stretch theories to nonlinear finite element analysis, Computers \& Structures 10 (1) (1979) 175-182. doi:dx.doi.org/10.1016/0045-7949(79)90085-3.

[12] M. A. Crisfield, A consistent co-rotational formulation for non-linear, threedimensional, beam-elements, Computer methods in applied mechanics and engineering 81 (2) (1990) 131-150. doi:10.1016/0045-7825(90) 90106-V.

[13] K.-J. Bathe, S. Bolourchi, Large displacement analysis of three-dimensional beam structures, International Journal for Numerical Methods in Engineering 14 (7) (1979) 961-986. doi:http://dx.doi.org/10.1002/nme.1620140703. 
[14] S. M. L. Adriaenssens, Stressed spline structures., Ph.D. thesis, University of Bath (2000).

[15] B. D'Amico, A. Kermani, H. Zhang, Form finding and structural analysis of actively bent timber grid shells, Engineering Structures. 81 (2014) 195-207. doi:http://dx.doi.org/10.1016/j.engstruct.2014.09.043.

[16] G. Senatore, D. Piker, Interactive real-time physics: An intuitive approach to form-finding and structural analysis for design and education, ComputerAided Design 61 (0) (2015) 32-41. doi: http://dx.doi.org/10.1016/j.cad. 2014.02 .007$.

[17] A. Day, An introduction to dynamic relaxation, The engineer 219 (1965) 218 221.

[18] J. R. H. Otter, A. C. Cassell, R. E. Hobbs, et al., Dynamic relaxation, in: ICE Proceedings, Vol. 35, Thomas Telford, 1966, pp. 633-656.

[19] P. Cundall, Explicit finite-difference method in geomechanics, Numerical Methods in Geomechanics, ASCE, (1976).

[20] B. Topping, A. Khan, Parallel computation schemes for dynamic relaxation, Engineering computations 11 (6) (1994) 513-548. doi:http://dx.doi.org/ $10.1108 / 02644409410799407$.

[21] W. Kutta, Beitrag zur näherungsweisen Integration totaler Differentialgleichungen, B.G Teubner, 1901.

[22] J. Porteous, A. Kermani, Structural timber design to Eurocode 5, 2nd edition, John Wiley \& Sons, 2013.

[23] BS EN 1995-1-1:2004+A1:2008. Eurocode 5: Design of timber structures Part 1-1: General - Common rules and rules for buildings, British Standards Institution.

[24] C. R. Calladine, Theory of shell structures, Cambridge university press, 1989.

[25] C. Williams, Generation of a class of structural forms for vaults and sails, The Structural Engineer 68 (12) (1990) 231-235. 
[26] A. Kilian, J. Ochsendorf, Particle-spring systems for structural form finding, Journal of the International Assossation for Shell and Spatial Structures 148 (2005) 77 .

[27] P. C. V. Block, Thrust network analysis: exploring three-dimensional equilibrium, Ph.D. thesis, Massachusetts Institute of Technology (2009).

[28] J. Harding, P. Shepherd, Structural form finding using zero-length springs with dynamic mass, in: 2011 IASS Annual Symposium: IABSE-IASS 2011: Taller, Longer, Lighter, University of Bath, 2011.

[29] P. Shepherd, W. Pearson, Topology optimisation of algorithmically generated space frames, in: Proc. of the IASS Symposium, Wroclaw, Poland, 2013.

[30] J. Thompson, G. W. Hunt, On the buckling and imperfection-sensitivity of arches with and without prestress, International Journal of Solids and Structures 19 (5) (1983) 445-459. doi:dx.doi.org/10.1016/0020-7683(83) 90055-0.

[31] S. P. Timoshenko, J. M. Gere, Theory of elastic stability, McGraw-Hill, 1961.

[32] EN ISO 527-1: 2012, Plastics - determination of tensile properties.

[33] S. A. Chini, A. M. Wolde-Tinsae, Effect of prestressing on elastica arches, Journal of engineering mechanics 114 (10) (1988) 1791-1800. doi:http:// dx.doi .org/10.1061/(ASCE) 0733-9399(1988)114:10(1791).

[34] A. Mirmiran, A. M. Amde, Effects of fabrication process on prestressed composite arches, (ASCE) Journal of Structural Engineering 121 (1) (1995) 124-131. doi:http://dx.doi.org/10.1061/(ASCE) 0733-9445(1995) 121: $1(124)$.

[35] P. Frieze, R. Hobbs, P. Dowling, Application of dynamic relaxation to the large deflection elasto-plastic analysis of plates, Computers \& Structures 8 (2) (1978) 301-310. doi : http://dx.doi .org/10 .1016/0045-7949 (78) 90037-8.

[36] L. Zhang, T. Yu, Modified adaptive dynamic relaxation method and its application to elastic-plastic bending and wrinkling of circular plates, Comput- 
ers \& structures 33 (2) (1989) 609-614. doi:http://dx.doi.org/10.1016/

[37] J. Natterer, N. Burger, A. Müller, Das expo-dach in hannover als pilotprojekt für den holzbau-entwicklung und einsatz nicht geregelter bauweisen, Bautechnik 78 (10) (2001) 693-705. doi:http://dx.doi.org/10.1002/ bate. 200104840 .

[38] J. Harding, W. Pearson, H. Lewis, S. Melville, The ongreening pavilion, in: Advances in Architectural Geometry 2014, Springer, 2015, pp. 295-308.

[39] S. Nabaei, O. Baverel, Y. Weinand, Form finding of twisted interlaced structures: a hybrid approach, in: Advances in Architectural Geometry 2014, Springer, 2015, pp. 127-143.

[40] T. Van Mele, L. De Laet, D. Veenendaal, M. Mollaert, P. Block, Shaping tension structures with actively bent linear elements, International Journal of Space Structures 28 (3) (2013) 127-136. doi:http://dx.doi.org/10.1260/ 0266-3511.28.3-4.127. 